\title{
Spirorchiidiasis in stranded loggerhead Caretta caretta and green turtles Chelonia mydas in Florida (USA): host pathology and significance
}

\author{
Brian A. Stacy ${ }^{1, *}$, Allen M. Foley ${ }^{2}$, Ellis Greiner ${ }^{3}$, Lawrence H. Herbst ${ }^{4}$, \\ Alan Bolten ${ }^{5}$, Paul Klein ${ }^{6}$, Charles A. Manire ${ }^{7}$, Elliott R. Jacobson ${ }^{1}$ \\ ${ }^{1}$ University of Florida, College of Veterinary Medicine, Large Animal Clinical Sciences, PO Box 100136, Gainesville, \\ Florida 32610, USA \\ ${ }^{2}$ Florida Fish and Wildlife Conservation Commission, Fish and Wildlife Research Institute, Jacksonville Field Laboratory, \\ 370 Zoo Parkway, Jacksonville, Florida 32221, USA \\ ${ }^{3}$ University of Florida, College of Veterinary Medicine, Infectious Diseases and Pathology, PO Box 110880, Gainesville, \\ Florida 32610, USA \\ ${ }^{4}$ Department of Pathology, Albert Einstein College of Medicine, Bronx, New York 10461, USA \\ ${ }^{5}$ Archie Carr Center for Sea Turtle Research, University of Florida, PO Box 118525, Gainesville, Florida 32611, USA \\ ${ }^{6}$ Department of Pathology, Immunology, and Laboratory Medicine, College of Medicine, University of Florida, Gainesville, \\ Florida 32610, USA \\ ${ }^{7}$ Mote Marine Laboratory and Aquarium, 1600 Ken Thompson Parkway, Sarasota, Florida 34236, USA
}

\begin{abstract}
Spirorchiid trematodes are implicated as an important cause of stranding and mortality in sea turtles worldwide. However, the impact of these parasites on sea turtle health is poorly understood due to biases in study populations and limited or missing data for some host species and regions, including the southeastern United States. We examined necropsy findings and parasitological data from 89 loggerhead Caretta caretta and 59 green turtles Chelonia mydas that were found dead or moribund (i.e. stranded) in Florida (USA) and evaluated the role of spirorchiidiasis in the cause of death. High prevalence of infection in the stranding population was observed, and most infections were regarded as incidental to the cause of death. Spirorchiidiasis was causal or contributory to death in some cases; however, notable host injury and/or large numbers of parasites were observed in some animals, including nutritionally robust turtles, with no apparent relationship to cause of death. New spirorchiid species records for the region were documented and identified genera included Neospirorchis, Hapalotrema, Carettacola, and Learedius. Parasites inhabited and were associated with injury and inflammation in a variety of anatomic locations, including large arteries, the central nervous system, endocrine organs, and the gastrointestinal tract. These findings provide essential information on the diversity of spirorchiids found in Florida sea turtles, as well as prevalence of infection and the spectrum of associated pathological lesions. Several areas of needed study are identified with regard to potential health implications in the turtle host, and findings caution against over-interpretation in individual cases.
\end{abstract}

KEY WORDS: Spirorchiidiasis $\cdot$ Trematode $\cdot$ Loggerhead sea turtle $\cdot$ Green sea turtle $\cdot$ Pathology Neospirorchis $\cdot$ Hapalotrema $\cdot$ Learedius

Resale or republication not permitted without written consent of the publisher

\section{INTRODUCTION}

Florida (USA) and its surrounding waters provide foraging and nesting habitat for loggerhead (Caretta caretta: CC) and green turtles (Chelonia mydas: CM). Approximately 1200 to 1500 strandings are docu- mented annually in Florida, primarily by the Sea Turtle Stranding and Salvage Network (STSSN; A. M. Foley et al. unpubl.). Health-related studies of sea turtles in Florida have included investigations of fibropapillomatosis, brevetoxicosis, hypothermic (cold)-stunning, and other mass mortality events (MMEs; Witherington 
\& Erhart 1989, Herbst 1994, Herbst et al. 2004, Foley et al. 2005, 2007, Jacobson et al. 2006). These studies have provided important insight, but also have revealed fundamental gaps in our knowledge of sea turtle diseases. Spirorchiid trematode infections in particular have been a recurring health concern in Florida sea turtles largely due to sporadic observations of heavily infected stranded turtles and an uncertain role in at least 1 MME (Wolke et al. 1982, Jacobson et al. 2006).

Sea turtles serve as definitive hosts for approximately 24 described species of spirorchiid trematodes representing 10 genera (Smith 1997a, Platt 2002). These numbers may be an underrepresentation, as all species and geographical ranges of sea turtles have not been thoroughly examined. Studies of spirorchiids in sea turtles tend to be polarized as either formal morphological descriptions and surveys of parasites or as descriptions of associated host pathology(e.g. Platt \& Blair 1998, Raidal et al 1998). Few studies have effectively combined specific identification of spirorchiid species, characterization of host pathology, and interpretation of the overall pathological significance of these parasites in the context of data from complete necropsies (but see Gordon et al. 1998). Furthermore, previous studies have been heavily biased towards chronically ill turtles, which often are diagnostically complex and require very conservative interpretation (Wolke et al. 1982, Gordon et al. 1998). As a result, the emergent understanding of spirorchiids and their impact on sea turtle health is very incomplete. Furthermore, basic information, such as prevalence of spirorchiids and associated pathological lesions in sea turtles found dead or moribund (i.e. stranded), is sparse or completely lacking for many regions, including the southeastern US.

The aim of this study was to better understand spirorchiidiasis and associated disease in stranded $\mathrm{CC}$ and $\mathrm{CM}$ by examining the following parameters: (1) the role of spirorchiids in the cause of death, (2) the diversity and prevalence of spirorchiids, (3) microhabitat utilization in the turtle host and associated pathological lesions. Additionally, relationships between parasitism, nutritional condition at stranding, and host age/size class were compared. The resulting findings provide the first comprehensive assessment of spirorchiidiasis in Florida sea turtles and offer new insight into host-parasite-disease relationships.

\section{MATERIALS AND METHODS}

Necropsies and parasitological methods. Necropsies were performed on stranded $\mathrm{CC}$ and $\mathrm{CM}$ recovered in Florida by the STSSN from November 2004 through January 2008. The study was limited to wild turtles that were found dead, died soon after discovery, or that were in rehabilitation centers for $10 \mathrm{~d}$ or less. Parasitological methods included a combination of gross examination, examination of target organs using a dissecting microscope, screening of body fluids and organ washes, and histopathology to detect and recover spirorchiid trematodes. The liver, body (coelomic) cavity, heart (and base of aortas and pulmonary arteries), aorta (left, right, and dorsal), and mesenteric arteries were examined and vascular compartments were thoroughly washed and filtered using a no. 45 sieve. In addition, the brain, heart, any vascular lesions, thyroid gland, thymus, adrenal glands, urinary bladder, and sections of stomach and intestine were examined under a dissecting microscope for spirorchiid eggs and adults. Four categories of necropsy and parasitological examination were performed depending on circumstances and postmortem condition. Category 1 necropsies included gross and histopathological examination of all organs and tissues, and complete parasitological methods for detection of spirorchiid trematodes. Category 2 necropsies included gross assessment, complete parasitological methods for detection of spirorchiid trematodes, and, in some cases, limited histopathology of major lesions. Category 3 necropsies consisted of gross necropsy and evaluation of the aortic system for endarteritis and examination of the brain, thyroid gland, adrenal glands, and thymus under a dissecting microscope. Category 4 examinations were limited to examination of the brain and meninges under a dissection microscope for the presence of spirorchiid trematodes. Categories 2 through 4 generally were performed on previously frozen and/or mildly autolyzed carcasses. The straight carapace length (SCL) was measured from the nuchal notch to the tip of the pygal and was used in categorization of size class. Samples for histological examination were fixed in $10 \%$ neutral phosphate buffered formalin and processed by routine methods into paraffin blocks, which were cut into $5 \mu \mathrm{m}$ thick sections and stained with hematoxylin and eosin.

Identification of spirorchiids was based on examination of adults whenever possible. When only eggs were found, their morphology alone was used to identify the genera Neospirorchis and Carettacola; however, egg morphology could not be used to distinguish Hapalotrema and Learedius egg types. Spirorchiid eggs in tissues were further characterized as primary site of oviposition or embolization to distant locations. Primary sites of oviposition were characterized by the presence of large and/or discrete egg masses ( $>100$ eggs) and were often observed in the presence of adult spirorchiids. In contrast, embolized eggs were diffusely distributed throughout a tissue or organ and were either individual eggs or small clusters of $<20$. Adult trematodes were fixed in alcohol-formalin-acetic 
acid (AFA) if intact or nearly complete, and representative specimens (including adults and eggs) were frozen at $-80^{\circ} \mathrm{C}$.

Nutritional condition at stranding. Nutritional condition at stranding was used as an indicator of duration of illness, and thus was a means of segregating turtles that had died from acute events from those with more chronic conditions. A single observer (B. A. Stacy) classified the nutritional condition of necropsied turtles as robust, mild to moderately underweight (intermediate), or poor based on the degree of atrophy of skeletal muscle and adipose tissue. Ideally, body weight would also have been compared; however, error in weight measurements due to variation between equipment, amount of epibiota, and possible loss of body mass in cases of severe traumatic injury prevented confident assessment of body condition indices (BCIs) in our entire study group. However, a group of immature CM that died from hypothermia presented the opportunity to relate BCIs to levels of parasitism and parasite-related pathology. Two BCIs were calculated, a simple mass to length ratio (body mass [BM; $\mathrm{kg}$ ] / straight carapace length [SCL; $\mathrm{Cm}$ ]) and estimated volume ratio $\left(\mathrm{BM} / \mathrm{SCL}^{3}\right)$, both of which have been used in previous sea turtle studies (Bjorndal et al. 2000, McMichael 2005, Work et al. 2005).

Primary diagnosis/cause of death designations. Necropsied turtles were also categorized by the primary diagnosis or proximate cause of death, if determined. Categories included traumatic injury, brevetoxicosis, hypothermia, MME of unknown etiology, drowning/aspiration, infectious disease, enteric impaction, fibropapillomatosis-associated, emaciation, multifactorial, and undetermined. The term primary diagnosis is used to indicate any notable necropsy finding when proximate cause of death could not be determined. For example, emaciation was listed as the primary diagnosis if poor nutritional condition was the only necropsy finding of significance, i.e. the underlying problem(s) was not apparent. Primary drowning/ aspiration cases were diagnoses by exclusion, were often accompanied by circumstantial evidence (e.g. recovered from trawler nets), and were not associated with recognized MMEs. Designations of brevetoxicosis, unsolved MMEs, and hypothermia were supported by epidemiological stranding data, environmental data, and supportive diagnostics from the investigations of these events (data not shown), rather than from specific pathological findings. Deaths were ruled multifactorial if multiple processes were present and were interpreted to have contributed to death. In every use, the latter designation reflected multiple health problems in chronically ill, emaciated turtles.

Spirorchiid impact rating (SIR) and assessment of parasitism/associated lesions. Spirorchiid trematode infection and associated pathological lesions were evaluated based on intensity of spirorchiid infection, severity of associated lesions, proportion of organ involvement, and the organ or tissue affected. All of these data were assessed in the context of other necropsy findings and cause of death, if determined, and used to assign turtles into 1 of 5 impact rating categories. The specific criteria for ratings are given in Appendix 1. Spirorchiid impact ratings were only applied to Category 1 and 2 necropsies.

Intensity of parasitism is classically measured by quantifying adult parasites and/or eggs, e.g. fecal egg counts. With regard to spirorchiid trematodes, the numbers of adult parasites often do not appear to correlate with severity of pathological lesions, and the intravascular location and small size of adults makes confident quantification extremely difficult or impossible (Gordon et al. 1998). Also, the variety of tissues in which eggs selectively accumulate makes any objective measure that would be comparable between cases very difficult. Therefore, the intensity of spirorchiidiasis in this study is based on a relative assessment of absent, rare, small, moderate, or large numbers, reflecting both the numbers of adults and eggs within various organs and tissues as observed at the gross and subgross levels. Photographs of cases and postmortem data were retrospectively compared to assure that abundance ratings were consistent within categories. In addition, specific grading criteria were created for vascular, neurological, thyroid/thymic, and gastrointestinal lesions as a measure of relative severity (Appendix 2).

Statistical methods. Comparisons were made between various aspects of spirorchiidiasis, including presence/ absence, pathological lesions, and grade, in turtles categorized by nutritional condition and size. Three size classes of sea turtles, $\leq 65 \mathrm{~cm},>65-85 \mathrm{~cm}$, and $>85 \mathrm{~cm}$, based on SCL (measured from the nuchal notch to the posterior marginal tip), were used. Parameters were compared by Pearson's chi-square or a Fisher exact test (95\% confidence interval), depending on the expected frequencies, using Analyse-it@ version 2.11. We also examined differences in BCIs in immature CM with and without moderate or large numbers of embolized spirorchiid eggs and intra-thyroid Neospirorchis species using the Mann-Whitney test.

\section{RESULTS}

\section{Caretta caretta}

Study population demographics and nutritional condition at stranding

A total of $89 \mathrm{CC}$ were necropsied. Carcasses were recovered from both Atlantic and Gulf coasts. The fre- 
quency distribution of turtle sizes was bimodal, with peak frequencies in the 60-75 and 85-95 cm SCL size ranges (Fig. 1). All turtles $>85 \mathrm{~cm}$ SCL were confirmed to be sexually mature based on examination of the reproductive tract. The female to male sex ratio was strongly biased at 3.9:1 (71:18). Category 1 and 2 necropsy data were obtained from 54 turtles: 20 nutritionally robust animals, 14 in the intermediate category, and 20 in poor nutritional condition. The numbers of turtles in each nutritional condition category by our 3 size classes were compared. The only statistical difference was that there was a higher proportion of turtles (Category 1 and 2 data) $>85 \mathrm{~cm}$ SCL in the nutritionally robust group than in poor condition $(p<0.05)$. The same bias was observed when all necropsy data categories (1-4) were analyzed $(\mathrm{p}<0.005)$.

\section{Primary diagnoses or causes of death by nutritional condition at stranding}

Primary diagnoses or causes of death of CC in Category 1 and 2 necropsies given by nutritional condition at stranding are shown in Table 1. Of those nutritionally robust animals, traumatic injuries were due to collisions with watercraft (2/6), falling from a sea wall while on the beach to nest (1/6), shark attack (1/6), a

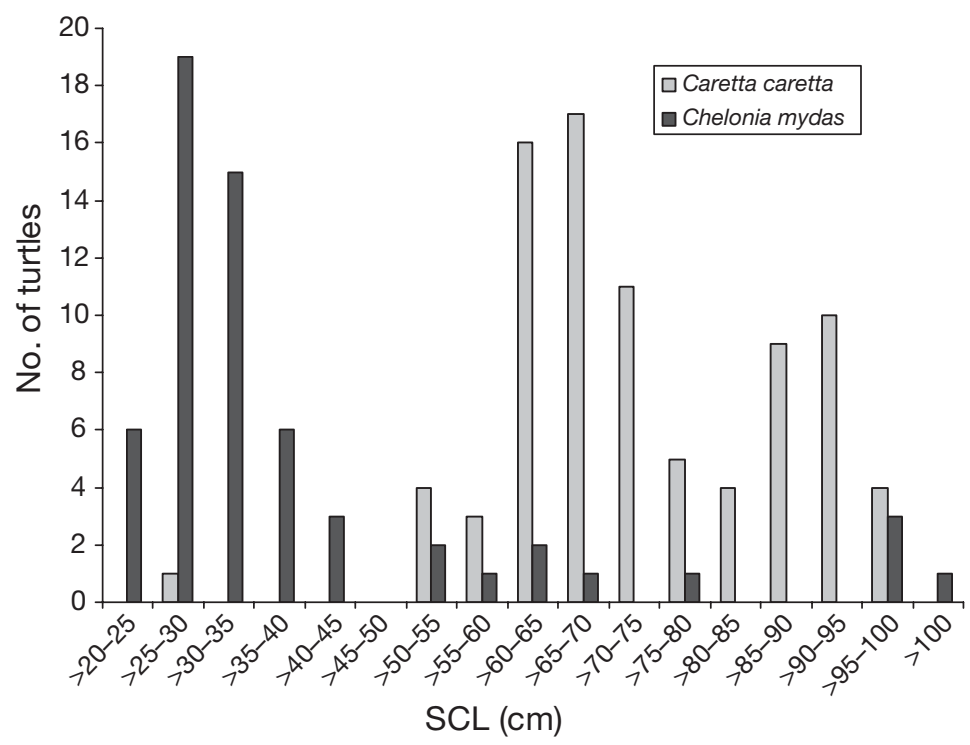

Fig. 1. Caretta caretta and Chelonia mydas. Size-class frequencies of necropsied turtles, by straight carapace length (SCL) probable hooking injury (1/6), and an unknown cause resulting in a fatal fracture (1/6). All brevetoxicosis cases were associated with a Karenia brevis bloom in southwestern Florida in 2005. The turtles that died during an MME of unknown etiology were all from the same event, which was documented along the coasts of northeastern Florida and Georgia in 2006. Of the remaining 3 turtles in good nutritional condition, 1 drowned in a trawler fishing net (1/20), 1 died of aspiration pneumonia due to unknown circumstances $(1 / 20)$, and the cause of death of a third case could not be determined $(1 / 20)$.

Of those $\mathrm{CC}$ in the intermediate condition category, 6/14 originated from the aforementioned 2006 MME. Five deaths associated with infectious disease or inflammatory conditions in this category were due to a variety of causes and affected systems. Cases included septic cholecystitis (1/5), fungal tracheobronchitis (1/5), chronic pneumonia of unknown etiology (1/5), necrotizing dermatitis (1/5), and septicemia with thromboembolic disease (1/5). The case of thromboembolic disease had atrial thrombi that formed in association with areas of intense endocardial spirorchiid egg deposition. Two deaths from traumatic injuries were both cases with chronic propeller wounds resulting in severe ascending bacterial meningitis.

Most CC in poor nutritional condition had multiple disease processes, and cause of death was classified as multifactorial (Table 1). No underlying inciting (primary) cause of disease could be confidently identified in most of these cases, nor was there any apparent association with known MMEs. Most postmortem findings 
in emaciated CC were regarded as either nonspecific lesions associated with chronic illness and poor nutritional status or as opportunistic (secondary) infections. In addition to being profoundly emaciated, common diagnoses interpreted to be contributory to death in these animals included ulcerative bacterial gastritis (8/13), ulcerative enteritis and/or colitis (7/13), dermatitis (most often associated with accumulation of epibiota) (8/13), granulomatous hepatitis (4/13), and cutaneous and/or musculoskeletal trauma associated with advanced catabolic state (2/13). In a few cases, emaciation alone was the most significant finding (3/20).

A single or predominant process was evident as an underlying condition or proximate cause of death in 4/20 emaciated CC. One turtle had severe chronic cardiovascular and gastroenteric spirorchiid-associated lesions that were interpreted as the cause of death. Two turtles had severe chronic colon impactions (comprised of bivalve shells) with associated ulcerative colitis. One turtle had chronic, obstructive inflammation of all major bile ducts (choledochitis) and subsequently drowned as a result of debilitation.

Prevalence by genus (Category 1 and 2 data)

The genera Neospirorchis, Hapalotrema, and Carettacola were identified in CC. In Category 1 and 2 necropsy data, period prevalence of Neospirorchis sp. was $96.3 \%$ (52/54), prevalence of Hapalotrema sp. was $77.8 \%(42 / 54)$, and prevalence of Carettacola sp. was $22.2 \%(12 / 54)$. Among infected CC, mixed infections by all 3 genera were observed in $21.2 \%$ (11/52), 2 genera in $61.5 \%(32 / 52)$, and infection by a single genus in $17.3 \%(9 / 52)$.

Identifiable spirorchiid species, site predilection, and associated pathological lesions

Hapalotrema species. Identification of Hapalotrema species was performed in 20 cases in which adult parasites were collected. Three species of Hapalotrema were found in CC, including $H$. mistroides (17/20), $H$. pambanensis (5/20), and a probable novel species $(2 / 20)$. Of those parasites identified to the species level, the sites from which adult spirorchiids were recovered, including the heart, aortas, mesenteric arteries, and hepatic vessels, are given in Table 2 . When categorized by nutritional condition at stranding (Category 1 and 2 necropsies), there were no detectable differences in numbers of CC infected with Haplotrema spp. However, more infected turtles were $>85 \mathrm{~cm}$ SCL than $\leq 65 \mathrm{~cm} \mathrm{SCL}(\mathrm{p}<0.05)$.
Table 2. Caretta caretta. Anatomic locations from which adult specimens of Hapalotrema species were recovered. Each data entry indicates the number of individual turtles from which a given species was recovered from a specific site, not the number of parasites

\begin{tabular}{|lcccc|}
\hline Species & Heart & Aortas & $\begin{array}{c}\text { Mesenteric } \\
\text { arteries }\end{array}$ & $\begin{array}{c}\text { Hepatic } \\
\text { vessels }\end{array}$ \\
\hline H. mistroides & 4 & 11 & 8 & 1 \\
H. pambanensis & 3 & 2 & 0 & 1 \\
$\begin{array}{c}\text { Hapalotrema sp. } \\
\text { (probable novel) }\end{array}$ & 0 & 0 & 2 & 1 \\
\hline
\end{tabular}

Endarteritis of major arteries was seen in $37.2 \%$ (29/78) of CC in all categories of necropsies. Affected vessels included the aortas and major branches. Pathological lesions were associated with Hapalotrema spp. based on the presence of parasites attached within areas of endarteritis in representative cases (Fig. 2A). Only 1 case had endarteritis with no evidence of Hapalotrema infection. Lesions in this case were minimal and chronic, which may reflect a resolved or mild infection. The association of a specific species with pathologic lesions is complicated because 4 of 20 turtles in which Hapalotrema spp. were identified had mixed infections including $H$. mistroides and $H$. pambanensis. However, H. mistroides was the only Hapalotrema sp. identified in all but $2 \mathrm{CC}$ with endarteritis. Also, neither of the 2 cases infected with the probable novel Hapalotrema species had arterial disease affecting larger arteries.

Categorization of large vessel endarteritis by grade, size class, and nutritional condition is given in Table 3. In Category 1 and 2 necropsies, the most common site of endarteritis/arteritis was the left aorta (25/29), followed by the superior mesenteric artery (11/29), right aorta (4/29), gastric artery (3/29), coeliac artery (1/29), and dorsal aorta (1/29). Multiple vessels were affected in 9/29 cases. Gross lesions ranged from single small plaques of endarteritis to larger areas of arteritis with associated generalized thickening of the artery wall (3 to $4 \mathrm{~mm}$; Fig. 2B) involving the entire left aorta and, in some cases, the superior mesenteric, coeliac, and gastric arteries. Mural thrombi were observed in 10 cases and all were non-occlusive. An aneurysm of the proximal superior mesenteric artery was present in a single turtle. Major arterial lesions were histologically examined in Category 1 necropsies ( $\mathrm{n}=24)$. Mild lesions (Grade 1) were characterized by mild subintimal fibrosis and a predominantly mononuclear inflammatory infiltrate. More severe lesions included variably intense pleocellular infiltrate, fibromuscular proliferation (often villous) of the intima (resulting in severe thickening of the artery wall), and degeneration and necrosis of the tunica media (Fig. 2C). The latter was a 

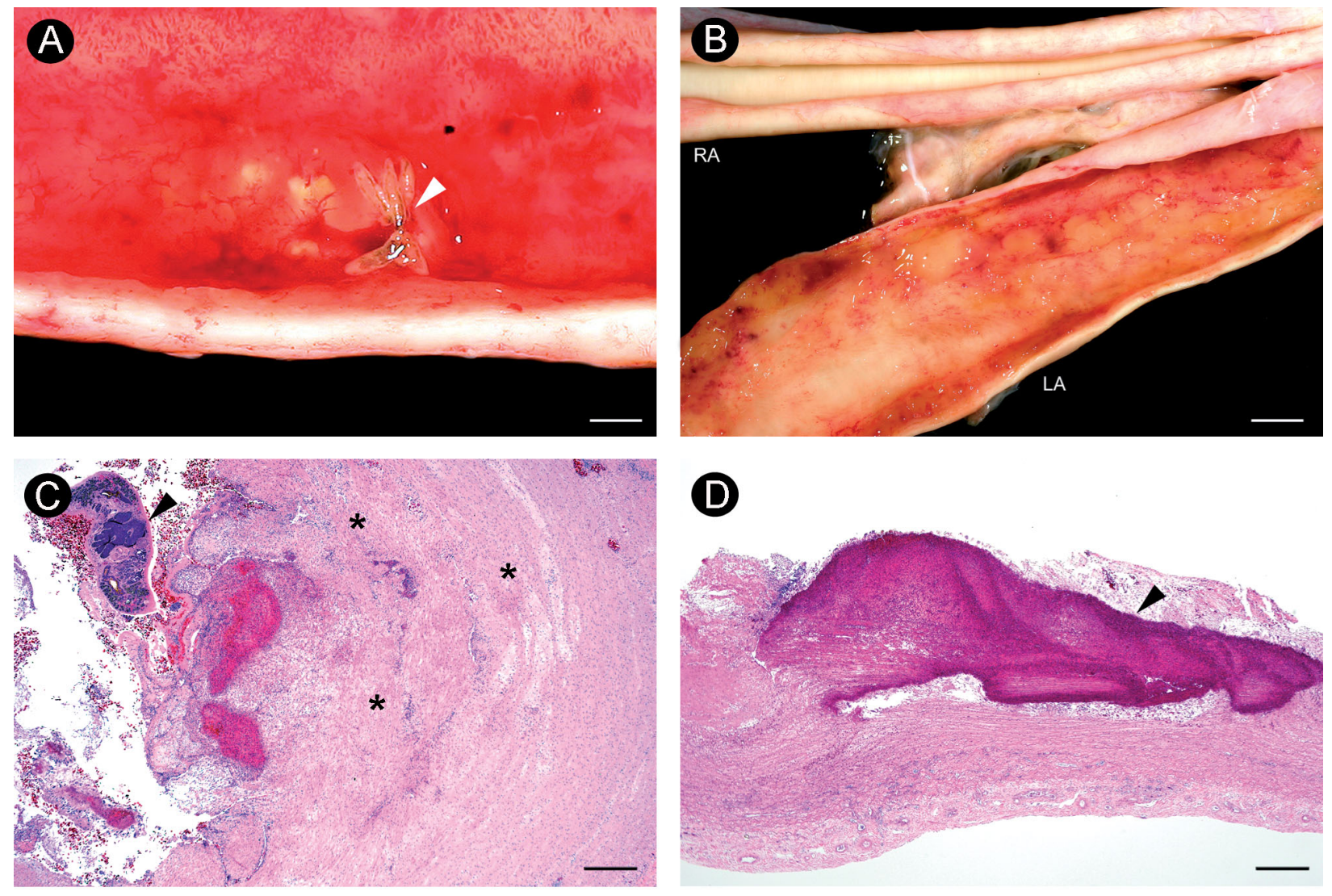

D
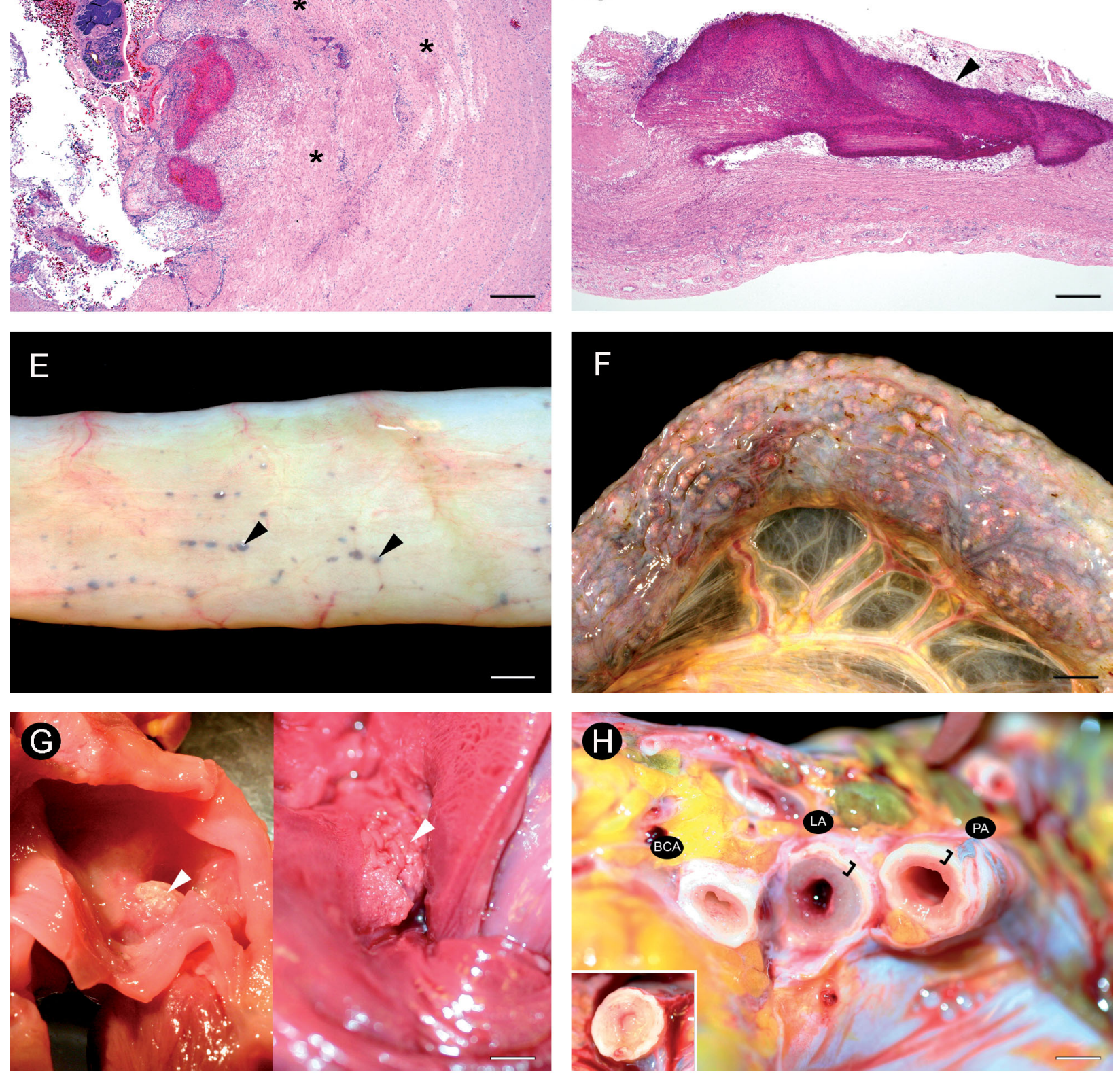
Table 3. Caretta caretta. Prevalence (\%, with number of cases/number of individuals examined in parentheses) of Hapalotrema-associated large vessel endarteritis by grade, nutritional condition at stranding, and size class. Grade 0: no lesions; Grade 1: minimal, focal $(<1.5 \mathrm{~cm}$ diameter) lesion; Grade 2: focally extensive ( 1.5 to $<4.0 \mathrm{~cm}$ diameter), moderate lesion; Grade 3: regionally extensive $(\geq 4.0 \mathrm{~cm})$ or diffuse lesion and/or with associated thrombus or aneurysm formation or necrosis of the tunica media. Nutritional condition at stranding based on presence/degree of atrophy of muscle and adipose stores

\begin{tabular}{|c|c|c|c|c|}
\hline & Grade 0 & Grade 1 & Grade 2 & Grade 3 \\
\hline \multicolumn{5}{|c|}{ Total $C$. caretta by grade of endarteritis } \\
\hline & $62.8(49 / 78)$ & $6.4(5 / 78)$ & $12.8(10 / 78)$ & $17.9(14 / 78)$ \\
\hline \multicolumn{5}{|c|}{ Nutritional condition } \\
\hline Robust & $64.0(16 / 25)$ & $12.0(3 / 25)$ & $16.0(4 / 25)$ & $8.0(2 / 25)$ \\
\hline Intermediate & $66.7(12 / 18)$ & $5.6(1 / 18)$ & $5.6(1 / 18)$ & $22.2(4 / 18)$ \\
\hline Poor & $60.0(21 / 35)$ & $2.9(1 / 35)$ & $14.3(5 / 35)$ & $22.9(8 / 35)$ \\
\hline \multicolumn{5}{|c|}{ Size class (straight carapace length, $\mathrm{cm}$ ) } \\
\hline$\leq 65$ & $66.7(14 / 21)$ & $9.5(2 / 21)$ & $9.5(2 / 21)$ & $14.3(3 / 21)$ \\
\hline$>65-85$ & $77.1(27 / 35)$ & $0(0 / 35)$ & $11.4(4 / 35)$ & $11.4(4 / 35)$ \\
\hline$>85$ & $14.3(2 / 14)$ & $14.3(2 / 14)$ & $21.4(3 / 14)$ & $50.0(7 / 14)$ \\
\hline
\end{tabular}

distinctive lesion characterized by presence of welldemarcated necrotic areas of smooth muscle surrounded and infiltrated by heterophils (Fig. 2D). This type of lesion was observed in 7 cases. Inflammation extended transmurally in 15 cases and often formed patchy or diffuse cuffs of intense pleocellular infiltrate within the tunica adventitia. Granuloma formation within lesions was common and most often included intralesional spirorchiid eggs, necrotic debris, and pigmented material.

No significant differences were detected in the prevalence or grade of endarteritis when CC were categorized by nutritional condition. However, there were again significant differences based on turtle size. More large turtles (>85 $\mathrm{cm} \mathrm{SCL}$ ) had endarteritis than either the $>65-85 \mathrm{~cm}$ or $\leq 65 \mathrm{~cm}$ SCL categories $(\mathrm{p}<0.005$ and $\mathrm{p}<0.0001$, respectively). There were no detect- able significant differences in grade of arteritis among infected turtles of the different size groups.

Pathological lesions affecting small arteries were histologically observed in the gastrointestinal tract and associated organs in $11 \mathrm{CC}$, all of which were infected by Hapalotrema species. Observed lesions included endarteritis, medial hypertrophy, microvascular proliferation, and periarteritis. Organs/ sites with affected vessels included the gastrointestinal tract $(\mathrm{n}=9)$, pancreas ( $\mathrm{n}=2)$, kidney $(\mathrm{n}=1)$, and adrenal gland ( $\mathrm{n}=1$ ). All but 2 turtles had concurrent endarteritis of major vessels and 6/9 had Grade 3 lesions. Some turtles had grossly visible Hapalotrema egg masses within subserosal vessels. Two distinct, grossly detectable variations of these egg masses were observed. The most common was characterized by small, raised, brown masses 2 to $3 \mathrm{~mm}$ in diameter (Fig. 2E). Most (6/7) of the turtles with this type of embolus had Grade 2 or 3 lesions in major arteries and were infected with $H$. mistroides or had mixed ( $H$. mistroides and $H$. pambanensis) infections. The less common variation of these egg masses was seen in a single turtle and was characterized by large transmural egg granulomas visible from the mucosal and serosal surfaces (Fig. 2F). This turtle did not have major arterial disease and was infected by the probable novel Hapalotrema species, adults and egg emboli of which were limited to the smaller arteries.

Six CC also had vasculitis of hepatic portal veins. All of these turtles were infected with Hapalotrema sp., but no adult spirochiids were recovered from the hepatic vasculature in any case. Two turtles were co-

Fig. 2. Caretta caretta and Chelonia mydas. Pathological lesions associated with Hapalotrema species (A-F) and Learedius learedi $(\mathrm{G}, \mathrm{H})$ infections in sea turtles. (A) C. caretta, left aorta. Five H. mistroides (white arrowhead) are attached to an area of endarteritis and the entire aortic wall is diffusely thickened (scale bar $=4.0 \mathrm{~mm}$ ). (B) C. caretta, aortas. The left aorta (LA) is diffusely thickened compared to the normal right aorta (RA) and includes multiple areas of endarteritis (scale bar $=0.7 \mathrm{~cm}$ ). $(\mathrm{C}) \mathrm{C}$. caretta, left aorta. Photomicrograph of the left aorta with severe endarteritis characterized by granulocytic inflammation (eosinophilic areas) and extensive fibromuscular proliferation (asterisks) corresponding to the arterial thickening present in the gross images. An H. mistroides (black arrowhead) is attached to the surface of the lesion (hematoxylin and eosin; scale bar $=400 \mu \mathrm{m}$ ). (D) C. caretta, left aorta. Photomicrograph of the left aorta with extensive necrosis of the tunica media (black arrowhead) associated with endarteritis. The hypereosinophilic character of the affected tissue is due to necrosis and infiltration by heterophils (hematoxylin and eosin; scale bar $=350 \mu \mathrm{m}$ ). (E) C. caretta, small intestine (serosal surface). Emboli of Hapalotrema eggs (black arrowheads) are visible within subserosal vessels. (F) C. caretta, small intestine (serosal surface; scale bar $=0.7 \mathrm{~cm}$ ). Another presentation of enteric Hapalotrema egg emboli characterized by formation of extensive indurated, transmural granulomas. This lesion was observed in association with a probable novel Hapalotrema species and resulted in severe ischemic necrosis of the mucosa (not grossly visible; scale bar $=1.0 \mathrm{~cm}$ ). $(\mathrm{G})$ C. mydas, heart (supravalvular region of atrium). Distinct raised areas of endarteritis are visible on the endocardium of 2 turtles (scale bar $\approx 0.6 \mathrm{~cm}$ ). $(\mathrm{H})$ C. mydas, major arteries. The brachiocephalic artery $(\mathrm{BCA}$ ), left aorta (LA), and pulmonary artery (PA) are severely thickened due to endarteritis. The white portion (tunica media) of the arterial wall (black brackets) represents the normal thickness of these arteries (scale bar $=0.7 \mathrm{~cm}$ ). The inset shows a severely stenotic vascular lumen within a more distal section through the BCA 
infected with Carettacola bipora, which were collected from the liver vasculature in 1 of these animals. Thus, it is possible that $C$. bipora is a cause of this lesion in some cases.

Papillary fibroepithelial lesions were seen in $2 \mathrm{CC}$ where submucosal granulomatous inflammation occurred in response to Hapalotrema eggs. One CC infected with $H$. mistroides had small colonic polyps. The other CC had severe granulomatous cholecystitis with papillary mucosal hyperplasia associated with the novel Hapalotrema species.

Neospirorchis species: general findings. In those $\mathrm{CC}$ infected with $\mathrm{Neo}$ spirorchis sp., trematode eggs typical of this genus were found widely embolized throughout the body. In addition, dense concentrations of eggs, often associated with adult parasites, were consistently found in the vasculature of the leptomeninges, endocrine organs, thymus, and submucosa of the alimentary tract. Specific species identification by morphology was not possible for most of the recovered Neospirorchis trematodes because suitable intact specimens were extremely difficult to collect. A diagnosis of Neospirorchis was based on egg morphology, elongate body type, and examination of anterior and posterior ends of some fragmented specimens. The only exception was $N$. pricei, which was easily recovered intact from the heart and major arteries. Prevalence of this species was $11.1 \%$ (6/54; Category 1 and 2 data). Genetic identification and characterization of Neospirorchis sp. was performed on most cases due to the limitations of morphological study and will be reported elsewhere.

Neospirorchis sp.: central nervous system. Adult Neospirorchis sp. and/or egg masses were observed in the leptomeninges of $51.7 \%(46 / 89)$ of the CC (all categories included; Table 4, Fig. 3A). Grading was performed in 45/46 infected turtles because a subdural hematoma prevented examination in 1 animal. The majority (29/45) of these cases were Grade 1 infections, although more than $10 \%$ (5/45) were classified as Grade 3. No significant differences were detected in either the proportions of turtles with neurological Neospirorchis infections or grades of infection when the study population was categorized by nutritional condition at stranding. However, significantly more infected CC were $>85 \mathrm{~cm}$ SCL than $>65-85 \mathrm{~cm}$ SCL $(p<0.01)$ or $\leq 65 \mathrm{~cm} \mathrm{SCL}(p<0.0001)$. There were no significant differences in grade among infected turtles when compared by size.

Adult Neospirorchis and egg masses were most commonly present within venules of the leptomeninges and surface vessels of the brain and spinal cord. Individual eggs and egg masses were both intravascular and perivascular. There was minimal or no inflammatory response associated with adult trematodes. Egg

Fig. 3. Caretta caretta and Chelonia mydas. Pathological lesions associated with Neospirorchis sp. infections in sea turtles. (A) C. caretta, brain. The brain is exposed revealing large numbers of Neospirorchis eggs within the leptomeninges (scale bar $=1.0 \mathrm{~cm}$ ). (B) C. caretta, heart (atrium). A large mural thrombus (white arrowhead) has formed over an area of intense Neospirorchis egg deposition in this turtle with thromboembolic disease (scale bar $=0.7 \mathrm{~cm}$ ). The eggs are more clearly visible in the high magnification inset. (C) C. mydas, thyroid gland. Large numbers of Neospirorchis eggs are distributed throughout the gland (scale bar $=850 \mu \mathrm{m}$ ). Tan areas of inflammation are associated with eggs and efface normal tissue. (D) C. caretta, thymus. Thymic vessels are distended by myriad Neospirorchis egg emboli, which obscure the thymus (scale bar $=9.0 \mathrm{~mm}$ ). Individual egg emboli are visible within a small vein in the inset. (E) C. caretta, small intestine (mucosal surface). Large numbers of black submucosal Neospirorchis egg masses are observed within mucosal folds (scale bar $=9.0 \mathrm{~mm}$ ). (F) C. caretta, colon. A closer image of an egg mass similar to those in (E) demonstrates a black serpiginous egg mass (asterisk) and resulting ulceration as eggs migrate into the lumen (scale bar $=3.5 \mathrm{~mm}$ ). An elongate yellow adult Neospirorchis (black arrowhead) is observed within a submucosal vessel. (G) C. mydas, lung. Moderate numbers of Neospirorchis eggs are embolized throughout the lung in this turtle that died of hypothermia (cold-stunning; scale bar $=600 \mu \mathrm{m}$ ). $(\mathrm{H})$ C. mydas, stomach (mucosal surface). Large numbers of Neospirorchis are diffusely embolized within the gastric submucosa. Multifocal tan areas of gastritis and ulceration (white arrowhead) are observed (scale bar $=850 \mu \mathrm{m})$ 

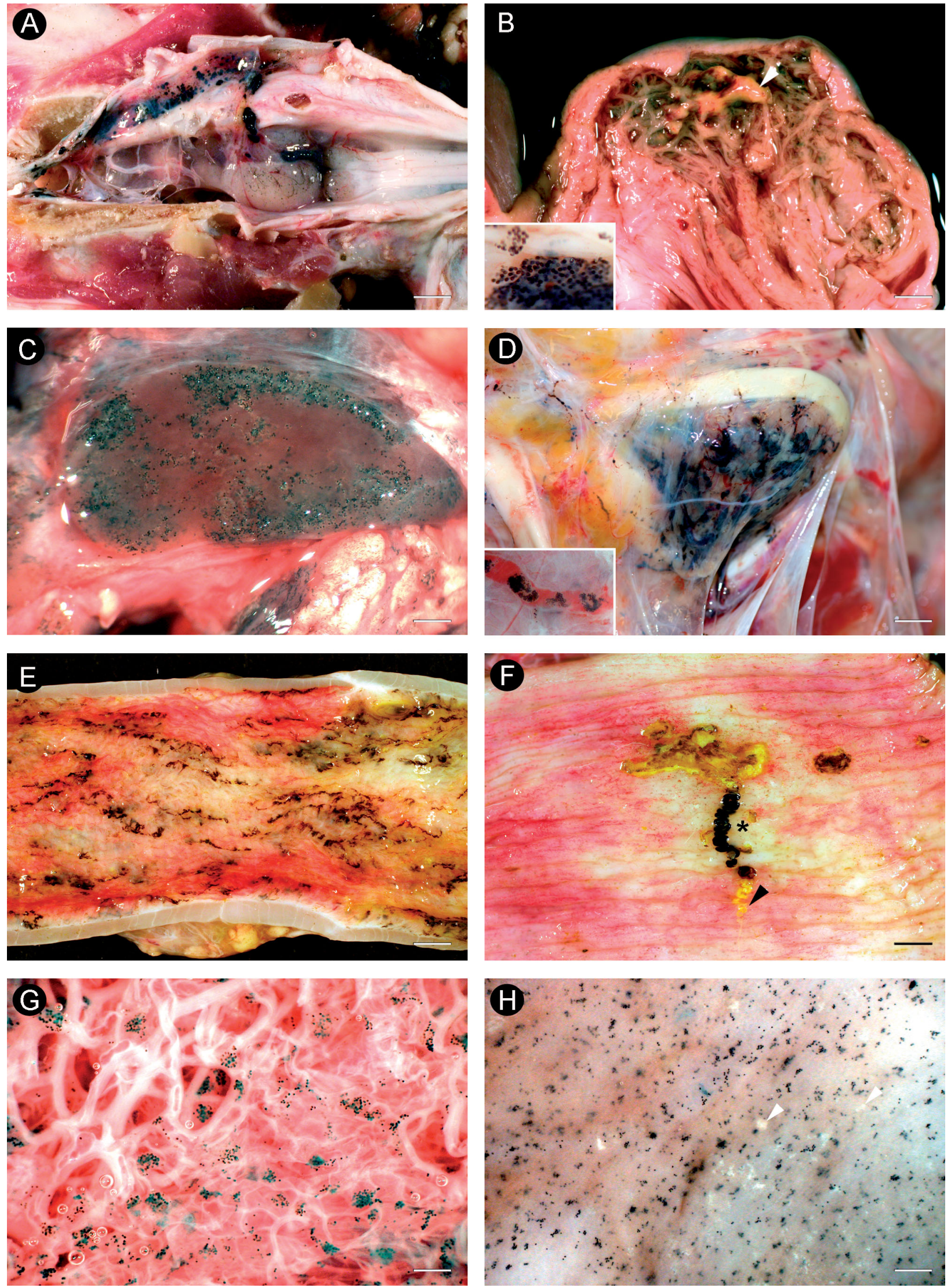
masses were often surrounded by granuloma formation, including intravascular granuloma formation, and a predominantly mononuclear infiltrate, resulting in meningitis and choroiditis. The severity of inflammation, with regard to distribution and intensity, generally corresponded to the number and distribution of eggs present in most cases. A fibrin thrombus was observed in a single case with Grade 3 infection. No associated ischemic or hemorrhagic lesions affecting the brain or spinal cord were observed in any infected CC. The ears were examined in some cases, and parasite presence was limited to mild egg embolization; however, complete histopathology of ears was not performed.

Neospirorchis sp.: heart and major arteries. All adult Neospirorchis sp. recovered from the heart and major arteries were identified as $N$. pricei. Of the infected turtles, 3 were emaciated and 3 were considered in intermediate nutritional condition. All infected CC were $>65 \mathrm{~cm}$ SCL. Most adult $N$. pricei were removed from the cardiac ventricle in all but 1 infected turtle. Small numbers of adults were also collected from the mesenteric vessels $(n=3)$, aortas $(n=1)$, and the carotid artery $(\mathrm{n}=1)$. One turtle infected with $N$. pricei died of septicemia and thromboembolic disease and had dense aggregates of eggs within the atria over which mural thrombi had formed (Fig. 3B).

Neospirorchis sp.: endocrine organs. The endocrine organ in which spirorchiid infection was most commonly observed was the thyroid gland (Fig. 3C). Of Category 1, 2, and 3 turtles, 54.6\% (41/75) had adult Neospirorchis sp. and/or eggs within the thyroid gland and $22.0 \%$ (9/41) of those infected were classified as Grade 3 (Table 5). Of Category 1 and 2 necropsies, where methods allowed careful detection of adult trematodes, $44.4 \%(24 / 54)$ had adult Neospirorchis observed within the thyroid gland. Significantly more emaciated CC had intrathyroidal spirorchiids as compared to animals in the nutritionally robust $(\mathrm{p}<0.005)$ and intermediate categories $(\mathrm{p}<0.05)$. Also, proportionately more emaciated $\mathrm{CC}$ had Grade 3 infections as compared to robust turtles $(p<0.05)$. No significant relationships between infection or grade of infection and turtle size were detected.

Severity of thyroid injury and associated inflammation generally was correlated with the numbers and distribution of eggs. In Grade 3 infections, the thyroid gland often appeared black on section due to the large numbers of spirorchiid eggs, which formed masses within the thyroid and surrounding cervical vasculature. Histologically, these lesions were characterized by an intense pleocellular infiltrate, granulomatous inflammation, thrombosis, and loss of thyroid follicles. Adult parasites, egg emboli, and vascular lesions, including medial hypertrophy and vasculitis, were observed in venules and arterioles.

Adult spirorchiids also were frequently observed in venules of the adrenal glands. Among Category 1 and 2 turtles, adult Neospirorchis sp. were observed in $32.0 \%(16 / 50)$. No egg masses were observed in this location, but individual embolized spirorchiid eggs were common (not included in period prevalence). Adrenalitis was minimal or mild in all cases and consisted of mild, mononuclear perivasculitis and eggassociated granulomas.

Adult Neospirorchis species were also observed included the pineal gland $(n=7)$. These observations were not included in any analyses due to small sample sizes.

Neospirorchis sp.: thymus. Another common site in which spirorchiids were observed in CC was the thymus, which was infected in $48.7 \%$ (37/76) of Category 1, 2, and $3 \mathrm{CC}$ (Table 6). The most commonly observed gross finding was egg masses within thymic vessels (Fig. 3D). All but 1 turtle with intrathymic spirorchiid eggs and/or adults also had intrathyroidal Neospirorchis. Of the thymic infections observed, $27.0 \%(10 / 37)$ were Grade 3 . When categorized by nutritional condition, emaciated CC were more likely to have thymic infections than robust turtles $(p<0.0001)$. Significant differences in the proportion of infected turtles were also detected when turtles were categorized by size. More $\mathrm{CC}$ in the $\leq 65 \mathrm{~cm}$ and $>65-85$ categories had thymic infections than turtles $>85 \mathrm{~cm}(\mathrm{p}<0.05)$. 
Table 6. Caretta caretta. Prevalence (\%, with number of cases/number of individuals examined in parentheses) of parasitism (Neospirorchis sp.) of the thymus by grade, nutritional condition, and size class. Grade 0: no parasites present; Grade 1: small numbers of egg masses, focal aggregates; Grade 2: moderate numbers of egg masses; Grade 3: large numbers of egg masses resulting is diffuse dark discoloration of organ. Nutritional condition at stranding based on presence/degree of atrophy of muscle and adipose stores

\begin{tabular}{|c|c|c|c|c|}
\hline & Grade 0 & Grade 1 & Grade 2 & Grade 3 \\
\hline \multicolumn{5}{|c|}{ Total $C$. caretta by grade of thymic spirorchiidiais } \\
\hline & $51.3(39 / 76)$ & $27.6(21 / 76)$ & $7.9(6 / 76)$ & $13.2(10 / 76)$ \\
\hline \multicolumn{5}{|c|}{ Nutritional condition } \\
\hline Robust & $84.0(21 / 25)$ & $16.0(4 / 25)$ & $0(0 / 25)$ & $0(0 / 25)$ \\
\hline Intermediate & $56.3(9 / 16)$ & $31.3(5 / 16)$ & $6.3(1 / 16)$ & $6.3(1 / 16)$ \\
\hline Poor & $25.7(9 / 35)$ & $34.3(12 / 35)$ & $14.3(5 / 35)$ & $25.7(9 / 35)$ \\
\hline \multicolumn{5}{|c|}{ Size class (straight carapace length, $\mathrm{cm}$ ) } \\
\hline$\leq 65$ & $40.0(8 / 20)$ & $25.0(5 / 20)$ & $15.0(3 / 20)$ & $20.0(4 / 20)$ \\
\hline$>65-85$ & $44.1(15 / 34)$ & $26.5(9 / 34)$ & $11.8(4 / 34)$ & $17.6(6 / 34)$ \\
\hline$>85$ & $78.6(11 / 14)$ & $21.4(3 / 14)$ & $0(0 / 14)$ & $0(0 / 14)$ \\
\hline
\end{tabular}

No significant relationships between grade of infection and duration of illness or size were detected.

Neospirorchis sp.: gastrointestinal tract. The prevalence data reported in this section include Neospirorchis species in which adults were found within the enterocolic submucosa and produced large black, serpiginous egg masses (typically 2.0 to $5.0 \mathrm{~cm}$ in length) that were visible from the mucosal surface (Fig. 3E). The enterocolic Neospirorchis sp. were found in $62.0 \%(31 / 50)$ of the CC (Table 7). A higher proportion of emaciated CC had Neospirorchis infections in the gastrointestinal tract than robust $\mathrm{CC}$ did $(\mathrm{p}<$ 0.005). No significant differences in grade of infection were detectable between nutritional condition categories. When categorized by size, there was a higher proportion of infected turtles in the >65-85 SCL size

Table 7. Caretta caretta. Prevalence (\%, with number of cases/number of individuals examined in parentheses) of enteric Neospirorchis sp. by grade, nutritional condition, and size class. Grade 0: no parasites present; Grade 1: 1 to 10 egg masses; Grade 2: $>10$ to 50 egg masses; Grade 3: $>50$ egg masses. Nutritional condition at stranding based on presence/degree of atrophy of muscle and adipose stores

\begin{tabular}{|c|c|c|c|c|}
\hline & Grade 0 & Grade 1 & Grade 2 & Grade 3 \\
\hline \multicolumn{5}{|c|}{ Total C. caretta by grade of enteric spirorchiidiasis } \\
\hline & $38.0(19 / 50)$ & $36.0(18 / 50)$ & $18.0(9 / 50)$ & $10.0(5 / 50)$ \\
\hline \multicolumn{5}{|c|}{ Nutritional condition } \\
\hline Robust & $66.7(12 / 18)$ & $22.2(4 / 18)$ & $11.1(2 / 18)$ & $0(0 / 18)$ \\
\hline Intermediate & $35.7(5 / 14)$ & $42.9(6 / 14)$ & $21.4(3 / 14)$ & $0(0 / 14)$ \\
\hline Poor & $11.1(2 / 18)$ & $44.4(8 / 18)$ & $22.2(4 / 18)$ & $22.2(4 / 18)$ \\
\hline \multicolumn{5}{|c|}{ Size class (straight carapace length, cm) } \\
\hline$\leq 65$ & $46.2(6 / 13)$ & $46.2(6 / 13)$ & $7.7(1 / 13)$ & $0(0 / 13)$ \\
\hline$>65-85$ & $20.0(3 / 15)$ & $20.0(3 / 15)$ & $40.0(6 / 15)$ & $20.0(3 / 15)$ \\
\hline$>85$ & $71.4(10 / 14)$ & $21.4(3 / 14)$ & $7.1(1 / 14)$ & $0(0 / 14)$ \\
\hline
\end{tabular}

group than in the $>85 \mathrm{~cm}$ size group $(p<0.01)$. No differences in grade of infection were observed among the different size groups.

Submucosal egg masses produced ulceration and secondary bacterial infection as eggs migrated through and injured the mucosa (Fig. 3F). Associated ulcerative enteritis was most severe inGrade 3 infections in which large areasof the mucosal surface were affected. Lesions were most common in the small intestine, where egg mass numbers and densities were the greatest.

Other findings related to Neospirorchis sp. In a few of the examined CCs, adult Neospirorchis were observed in several additional sites either by organ lavage, subgross examination, or histological examination. The most extensive tissue injury was observed in the testes of 3 $\mathrm{CC}$ in which adult Neospirorchis were present and abundant eggs were seen associated with granulomatous inflammation. A severe associated orchitis was present in 1 case, and an associated orchitis of more moderate severity was observed in the other 2 cases.

Of the Category $1 \mathrm{CCs}$, several specimens of $\mathrm{Neo}$ spirorchis species were flushed from the hepatic vasculature of $1 \mathrm{CC}$, and microscopic trematodes, consistent in morphology with Neospirorchis sp., were observed in the sinusoids of 3 additional turtles. No associated inflammatory response in the liver was observed. Additional locations where intravascular adult Neospirorchis were observed included single cases and rare or individual parasites. Sites included the lungs, nasal submucosa, arterial periadventitia, and bile duct periadventitia.

Carettacola species. All adult Carettacola were identified as C. bipora and all were removed from hepatic vessels. As previously mentioned, vasculitis of hepatic vessels was present in 2 infected turtles; however, adults and eggs of $C$. bipora were found only in relatively low numbers in all cases and with no direct association with any grossly visible lesions.

\section{Spirorchiid impact rating (SIR)}

The relationship of spirorchiid infections to the cause of death in most CC was determined to be either incidental $(27 / 54)$ or undetermined (18/54). 
Spirorchiid infections in a smaller number of cases $(6 / 54)$ were determined to be contributory to the cause of death. In $1 \mathrm{CC}$, spirorchiid infection was believed to be directly responsible for death, and no spirorchiid infections were detected in the remaining 2 cases (Table 8). Turtles were placed into 2 groups: those uninfected or with incidental infections (SIR 1 and 2) and those with contributory, fatal or undetermined impact ratings (SIR 3, 4, and 5, respectively; reflecting relatively heavy parasitism and spirorchiid-associated lesions). When compared by nutritional condition at stranding, significantly more nutritionally robust CC were in the uninfected and incidental group as compared to emaciated CC ( $p<0.005)$. No significant differences were detected when the same comparisons were made among size classes.

Common arrays of findings were reflected in the criteria for categorizing the significance of spirorchiid infections as contributory, fatal, or undetermined. Within the contributory category, 5/6 CC were emaciated and had multi-organ lesions. The key determining factor for assigning a SIR of contributory, as stated in the scoring criteria, was the presence of significant multisystemic lesions associated with eggs or adult parasites. Of these cases, 1 turtle had extensive ulceration of the pylorus as a primary necropsy finding that was associated with submucosal embolization of Hapalotrema eggs. Severe arteritis (H. mistroides) was observed in 4 turtles, thyroiditis (Neospirorchis sp.) in 2 cases (1 of which also had thymitis), and 2 cases had abundant (Grade 3) meningeal spirorchiids (Neospirorchis sp.). Considerable embolization of eggs was interpreted as contributory in 3 cases in which abundant eggs were found in the gastroenteric submucosa and lungs. One case also had abundant enteric Neospirorchis egg masses with associated enteritis, and another had severe orchitis (Neospirorchis sp.). The only non-emaciated turtle in the contributory cat-

Table 8. Caretta caretta. Spirorchiid impact ratings (SIR) categorized by nutritional condition at stranding. SIR 1: no spirorchiids detected; SIR 2: incidental infection - low numbers and mild if any associated host injury/response; SIR 3: contributory - spirorchiidiasis was one of multiple findings interpreted to have contributed to death; SIR 4: fatalspirorchiidiasis cause of death; SIR 5: unknown - relationship/ significance to cause of death undetermined. Nutritional condition at stranding based on presence/degree of atrophy of muscle and adipose stores

\begin{tabular}{|lccccc|}
\hline & SIR 1 & SIR 2 & SIR 3 & SIR 4 & SIR 5 \\
\hline Robust & 1 & 15 & 0 & 0 & 4 \\
Intermediate & 1 & 6 & 1 & 0 & 6 \\
Poor & 0 & 6 & 5 & 1 & 8 \\
Total & 2 & 27 & 6 & 1 & 18 \\
\hline
\end{tabular}

egory was an underweight animal with septicemia and thromboembolic disease. In this case, atrial thrombi formed over areas of intense endocardial Neospirorchis ( $N$. pricei) egg deposition.

The only case in which spirorchiidiasis could be confidently confirmed as the cause of death was in a CC with massive egg granulomas in the gastrointestinal tract, liver, gall bladder, and other organs associated with a probable novel Hapalotrema sp. The extent of spirorchiid egg granulomas in this case resulted in secondary ischemia of the enteric mucosa, ulceration, and probable septicemia.

Of those cases in which the impact of spirorchiidiasis was undetermined, $44.4 \%(8 / 18)$ were emaciated and $55.6 \%(10 / 18)$ were in the robust or intermediate nutritional condition categories. As stated in the scoring criteria, all had identifiable causes of death or multiple findings that could not be confidently associated with spirorchiidiasis based on lesion location and disease process or severity. For example, the emaciated CC had an identifiable proximate cause of death or contributing health problems, but did not have the multisystemic lesions comparable to those given a contributory rating. The most common spirorchiid associated lesions in animals in the undetermined category was Grade 3 arteritis (12/18), neurospirorchiidiasis (6/18), and thyroiditis (6/18). Of these animals, 11/18 had lesions involving more than 1 organ or system, typically arteritis and neurospirchiidiasis or thyroiditis. Findings represented by individual cases included 1 turtle with large numbers of Neospirorchis pricei in the heart, 1 animal with severe thymitis (concurrent with thyroiditis), and 1 animal with large numbers of eggs embolized in the enteric mucosa.

\section{Chelonia mydas}

\section{Study population demographics and nutritional condition categories}

Fifty-nine CM were examined. The study population was heavily biased toward smaller, immature animals $<45 \mathrm{~cm}$ SCL (Fig. 1). The female to male ratio was 2:1 (38/19; data unavailable for 3 turtles). Category 1 and 2 data included 50 turtles, of which 37 were nutritionally robust, 8 were in intermediate condition, and 5 were emaciated (Table 9). The large number of turtles in the robust category reflected 30 animals that stranded during a cold-stunning event in St. Joseph Bay in January 2008. In addition, all turtles $>65 \mathrm{~cm}$ SCL fell within the robust category, thus all CM in intermediate condition or emaciated were comprised completely of turtles $\leq 65 \mathrm{~cm}$ SCL. 
Table 9. Chelonia mydas. Causes of death categorized by nutritional condition at stranding for turtles examined by complete necropsy and inclusive spirorchiid trematode recovery methods (Category 1 and 2 data). Nutritional condition at stranding based on presence/degree of atrophy of muscle and adipose stores

\begin{tabular}{|lcccc|}
\hline $\begin{array}{l}\text { Primary diagnosis/ } \\
\text { cause of death }\end{array}$ & Robust & $\begin{array}{c}\text { Inter- } \\
\text { mediate }\end{array}$ & Poor & Total \\
\hline Hypothermic stunning & 30 & 0 & 0 & 30 \\
Traumatic injury & 7 & 3 & 1 & 11 \\
Fibropapillomatosis & 0 & 2 & 1 & 3 \\
Infectious disease & 0 & 3 & 1 & 4 \\
Emaciation & 0 & 0 & 1 & 1 \\
Multifactorial & 0 & 0 & 1 & 1 \\
Total & 37 & 8 & 5 & 50 \\
\hline
\end{tabular}

Primary diagnosis or cause of death by nutritional condition at stranding

The deaths of all CM in robust nutritional condition were due to hypothermia (cold-stunning) (30/37) and traumatic injuries (7/37; Table 9$)$. All of the hypothermia cases originated from a single event and all turtles had been actively foraging as indicated by abundant seagrass within the stomach. Most of the traumatic injuries (6/7) were watercraft-related. A seventh turtle was a nesting female that died from suffocation after a dune collapsed on her while nesting (1/6). Turtles in intermediate nutritional condition included CM with traumatic injuries (3/8), infectious diseases/inflammatory conditions (3/8), and fibropapillomatosis (2/8). Two turtles with traumatic injuries had watercraft-related injuries with secondary bacterial infection (2/3), and 1 had a chronic, strangulating entanglement wound on its neck (1/3). Infectious diseases and inflammatory conditions included fungal pneumonia (1/3), fungal tracheitis (1/3), and probable septicemia (1/3). Of the 2 turtles in which fibropapillomatosis was the primary finding, 1 was euthanized due to the presence of visceral tumors, and 1 had a severe marine leech infestation (Ozobranchus sp.) and anemia. Observations in emaciated CM were varied and included bacterial meningoencephalitis (1/5), fibropapillomatosis (1/5), a chronic entanglement wound (1/5), emaciation (1/5), and multifactorial (1/5).

Prevalence by genus (Category 1 and 2 data)

Three genera of spirorchiid trematodes were found in examined CM, including Neospirorchis in $92.0 \%$ (46/50), Learedius in $8.0 \%$ (4/50), and Hapalotrema in $2.0 \%(1 / 50)$. Type I eggs (Hapalotrema sp. or Leare- dius sp.) were observed in 2 additional turtles from which no adult parasites were recovered. Among infected CM, the majority (45/50) were infected by 1 genus, typically Neospirorchis, and 5 were infected by 2 different genera.

Identifiable spirorchiid species, site predilection, and associated pathological lesions

Learedius learedi. All Learedius specimens were identified as L. learedi. Sites from which L. learedi were recovered included the heart and hepatic vessels. Three of the 4 infected CM were adults and 1 was an immature animal with an SCL of $25.6 \mathrm{~cm}$. Thus, only 1 immature turtle had evidence of $L$. learedi infection out of 46 immature turtles examined (Category 1 and 2 data). The 3 adult turtles died from traumatic injuries (robust nutritional condition), and the juvenile was underweight and had fungal pneumonia (intermediate category). Two of the adult CM had associated distinct, focally extensive endocarditis located supravalvular to the atrioventricular valves (Fig. 2G). These lesions were observed grossly as well-demarcated, raised, tan-pink areas with an irregular surface and histologically were characterized by a mixed mononuclear and granulocytic infiltrate, fibrosis, and surface villous proliferation. Milder, more diffuse endocarditis was also present in both turtles. In addition, both animals with endocardial lesions also had severe thickening of the aortic system due to marked subintimal proliferation and mild pleocellular endarteritis. The extent of these lesions in 1 case was more extensive and involved the pulmonary, brachiocephalic, carotid, mesenteric, and associated smaller arteries (Fig. $2 \mathrm{H}$ ), as well as prominent generalized thickening/hypertrophy of the myocardium.

Hapalotrema postorchis. Only $1 \mathrm{CM}$ was confirmed to be infected by a species of Hapalotrema, which was identified as $H$. postorchis. The infected turtle was an adult male that died as the result of head trauma sustained during entrapment in a coastal power facility. The aortic system was prominently thickened, which reflected chronic arteritis similar to that observed in turtles infected with Learedius learedi. The left aorta was most severely affected and had multifocal, grossly visible plaques of endarteritis containing several embedded $H$. postorchis. Lesions in small arteries, including the mesenteric, renal, adrenal, and carotid arteries, were also observed.

Neospirorchis sp. As in CC, specific species identification of Neospirorchis specimens was not possible due to the inability to obtain intact parasites suitable for identification. Genetic characterization of collected specimens will be reported elsewhere. Spirorchiid 
eggs consistent in morphology with Neospirorchis sp. were observed embolized throughout the body and were most readily visible in the lung and submucosa of the gastrointestinal tract. In addition, dense concentrations of eggs and/or adult parasites were found in some locations similar to that observed in $\mathrm{CC}$, specifically the central nervous system, endocrine organs, and rarely within the gastric submucosa.

Neospirorchis sp.: central nervous system. Adult spirorchiids and/or egg masses identified as Neospirorchis sp. were found in leptomeninges of $11.9 \%$ of necropsied CM (7/59). These findings must be qualified with regard to grade and size class considerations. Foremost, all egg masses tended to be very small (often $1 \mathrm{~mm}$ or less), thus the criteria used to quantify/grade CC infections were scaled by abundance of eggs, rather than egg mass size to represent equivalent abundance in CM. Second, adult parasites were only observed in subadult or mature $\mathrm{CM}$, which included $5 / 7$ infected turtles. Four were adults with SCL of $95 \mathrm{~cm}$ or greater that died from acute traumatic events. The fifth turtle was submitted as a head only and based on its size was estimated to be a large subadult or adult. Thus, all of the mature CM examined had neurological infections. Two cases were classified as Grade 1, and 3 cases as Grade 2. The remaining 2 turtles were immature, one of unknown size and another with a SCL of $43.1 \mathrm{~cm}$. In both cases, no adult Neospirorchis were observed, and egg masses consisted of very small numbers of eggs (both Grade 1), which may reflect embolization rather than oviposition within meningeal vessels, as is apparent when adult parasites are present. Nevertheless, these cases were included because similar patterns of egg embolization (from dis-

Table 10. Chelonia mydas. Prevalence (\%, with number of cases/number of individuals examined in parentheses) of thyroid gland parasitism (Neospirorchis sp.) by grade, nutritional condition at stranding, and size class. Grade 0: no parasites present; Grade 1: small numbers of adults/eggs, focal aggregates; Grade 2: moderate numbers of eggs/adults, including focally extensive or diffuse aggregates; Grade 3: large numbers of eggs/adults with effacement of large areas of gland by parasites and associated inflammation. Nutritional condition at stranding based on presence/degree of atrophy of muscle and adipose stores

\begin{tabular}{|c|c|c|c|c|}
\hline & Grade 0 & Grade 1 & Grade 2 & Grade 3 \\
\hline \multicolumn{5}{|c|}{ Total C. mydas by grade of thyroid spirorchiidiasis } \\
\hline & $66.7(34 / 51)$ & $15.7(8 / 51)$ & $11.8(6 / 51)$ & $5.9(3 / 51)$ \\
\hline \multicolumn{5}{|c|}{ Nutritional condition } \\
\hline Robust & $57.9(22 / 38)$ & $21.0(8 / 38)$ & $15.8(6 / 38)$ & $5.3(2 / 38)$ \\
\hline Intermediate & $100(8 / 8)$ & $0(0 / 8)$ & $0(0 / 8)$ & $0(0 / 8)$ \\
\hline Poor & $80.0(4 / 5)$ & $0(0 / 5)$ & $0(0 / 5)$ & $20.0(1 / 5)$ \\
\hline \multicolumn{5}{|c|}{ Size class (straight carapace length, $\mathrm{cm}$ ) } \\
\hline$\leq 65$ & $63.8(30 / 47)$ & $17.0(8 / 47)$ & $10.6(5 / 47)$ & $8.5(4 / 47)$ \\
\hline$>65-85$ & $100(1 / 1)$ & $48.5(0 / 1)$ & $27.3(0 / 1)$ & $18.2(0 / 1)$ \\
\hline$>85$ & $75(3 / 4)$ & $0(0 / 0)$ & $25.0(1 / 4)$ & $0(0 / 0)$ \\
\hline
\end{tabular}

tant sites) were not observed in the other 52 turtles examined.

Neospirorchis sp.: endocrine organs. Spirorchiid parasitism of the thyroid gland was observed in $33.3 \%$ $(17 / 51)$ of necropsied CM (Table 10). Of the infected turtles, adult parasites were observed in $58.8 \%$ $(10 / 17) ; 17.6 \%(3 / 17)$ were classified as Grade 3 infections (Fig. 3C). Comparisons with other factors were limited given the biases of the study population and the limited number of emaciated, mature, and large immature turtles examined. Associated pathological lesions were as described for CC.

Adult parasites were recovered from the pineal gland in 2 turtles, and eggs were focally concentrated at this site in a third case. One turtle was found to have adult Neospirorchis sp. in the parathyroid gland. Findings in other endocrine organs were limited to embolization of eggs. No adult parasites were observed in the adrenal glands

Neospirorchis sp.: gastrointestinal tract. The only spirorchiid-related necropsy finding in the alimentary tract that exhibited a distinct pattern, other than widespread or regionally intense egg embolization, was the finding of small egg masses within the gastric submucosa. Egg deposition at this location was observed in $10.4 \%$ (5/48) of examined CM, which were all immature and represented all nutritional condition categories. This finding typically was only visible by examination of the gastric mucosa under a dissecting microscope, and associated inflammatory lesions were minimal or mild. No adult spirorchiids were observed grossly, but were seen histologically in 2 cases. In an additional case, similar eggs and a single adult spirorchiid were observed in the small intestine by histological examination. Other than this example, no discrete enteric Neospirorchis egg masses, as observed in $\mathrm{CC}$, were seen in CM.

Other findings related to Neospirorchis sp. In addition to finding adult spirorchiids and egg masses in the above anatomical locations, widespread egg embolization in relatively high densities were observed in 7 cases, all but one of which were immature turtles in robust nutritional condition. Eggs were most readily observed in the lungs and gastrointestinal mucosa of 4 cases, primarily the lungs in 2 cases, and primarily the gastrointestinal tract in 1 case (Fig. 3G,H). Adult parasites and/or egg masses in these cases were limited to the thyroid gland, which was parasitized in 4 turtles. 
Table 11. Chelonia mydas. Spirorchiid impact ratings (SIR) categorized by nutritional condition at stranding. SIR 1: no spirorchiids detected; SIR 2: incidental infection - low numbers and mild if any associated host injury/response; SIR 3: contributory - spirorchiidiasis one of multiple findings interpreted to have contributed to death; SIR 4: fatal- spirorchiidiasis cause of death; SIR 5: unknown-relationship/ significance to cause of death undetermined. Nutritional condition at stranding based on presence/degree of atrophy of muscle and adipose stores

\begin{tabular}{|lccccc|}
\hline & SIR 1 & SIR 2 & SIR 3 & SIR 4 & SIR 5 \\
\hline Robust & 4 & 24 & 0 & 0 & 9 \\
Intermediate & 0 & 7 & 1 & 0 & 0 \\
Poor & 0 & 4 & 0 & 0 & 1 \\
Total & 4 & 35 & 1 & 0 & 10 \\
\hline
\end{tabular}

\section{Spirorchiid impact rating}

The relationship of most spirorchiid infections to the cause of death in CM was determined to be incidental (35/50), followed by undetermined (10/50) and contributory (1/50). Four turtles were uninfected, and spirorchiid infection was not identified as the cause of death in any CM (Table 11). The biases in size class representation of $\mathrm{CM}$ and nutritional condition at stranding prevented comparison of rating within these categories.

Three findings of concern were observed in CM where the relationship of the spirorchiid infection to the cause of death could not be determined. These were cardiovascular disease associated with Learedius learedi and Hapalotrema postorchis in adult turtles ( $\mathrm{n}=3$ ), severe (Grade 3) parasitism (by Neospirorchis sp.) of the thyroid gland in immature turtles ( $\mathrm{n}=3$ ), and intense egg embolization (of Neospirorchis sp.) in the lungs and/or gastrointestinal tract of immature turtles $(\mathrm{n}=4)$. Three of the 4 latter cases also had Grade 2 thyroid parasitism. The 3 adult turtles were all males that died of acute traumatic injuries. Six of the immature turtles died from hypothermic stunning. The tenth case was an emaci- ated immature turtle with severe fibropapillomatosis and presumptive septicemia.

The single turtle in which spirorchiidiasis was interpreted as contributory to death had massive numbers of Neospirorchis eggs within the pulmonary vasculature and severe fibropapillomatosis. The lungs in this case were grossly discolored due to the large numbers of spirorchiid eggs.

\section{Body condition indices in hypothermic-stunned immature CM}

BCIs were calculated for the $30 \mathrm{CM}$ that died of hypothermia (Table 12). No significant differences were observed in BCIs, using either calculation, when turtles were compared based on the presence/absence of Neospirorchis in the thyroid gland or presence of relatively large numbers of Neospirorchis eggs concentrated in one or more organ systems. The latter group primarily included turtles with large numbers of intra-thyroidal eggs and moderate to large numbers embolized to the lungs and alimentary submucosa. The only significant difference was that CM with intrathyroidal Neospirorchis had a slightly greater SCL, although there was considerable overlap in sizes between infected and uninfected turtles ( $p<0.05)$.

\section{DISCUSSION}

The study populations of CC and CM reflect the predominant size classes and biased sex ratios of each species represented in the historical stranding data for Florida (A. M. Foley et al. unpubl.). However, several factors must be considered in any extrapolation of findings of this study to all stranded sea turtles. The goal of necropsy efforts was not to mimic actual demographic proportions of all strandings, but to include representation of major size classes to gain a more complete understanding of the host-parasite relationship. In addition, turtles that died from acute insults and were

Table 12. Chelonia mydas. Median values and ranges of straight carapace length (SCL [cm], measured from the nuchal notch to the posterior marginal tip) and body condition indices (BCIs) of hypothemic(cold)-stunned immature turtles with and without intrathyroidal Neospirorchis sp. and moderate to large numbers of diffusely embolized Neospirorchis eggs. Two BCIs were calculated from body mass $(\mathrm{BM}, \mathrm{kg})$ : estimated volume ratio $\left(\mathrm{BCI}_{1}=\mathrm{BM} / \mathrm{SCL}^{3}\right)$ and a simple mass to length ratio $\left(\mathrm{BCI}_{2}=\mathrm{BM} / \mathrm{SCL}\right)$

\begin{tabular}{|c|c|c|c|c|c|c|}
\hline \multirow[t]{2}{*}{ Chelonia mydas groups } & \multicolumn{2}{|c|}{$\mathrm{SCL}(\mathrm{cm})$} & \multicolumn{2}{|c|}{$\mathrm{BCI}_{1}\left(\mathrm{~kg} \mathrm{~cm}^{-3}\right)$} & \multicolumn{2}{|c|}{$\mathrm{BCI}_{2}\left(\mathrm{~kg} \mathrm{~cm}^{-1}\right)$} \\
\hline & Median & Range & Median & Range & Median & Range \\
\hline With intrathyroidal Neospirorchis & 31.5 & $25.9-33.7$ & 1.17 & $1.10-1.34$ & 1.18 & $0.58-1.54$ \\
\hline Without intrathyroidal Neospirorchis & 29.0 & $28.1-31.0$ & 1.18 & $1.11-1.33$ & 1.05 & $0.61-1.26$ \\
\hline With abundant embolized Neospirorchis eggs & 30.0 & $22.4-33.1$ & 1.17 & $1.13-1.30$ & 1.06 & $0.58-1.42$ \\
\hline Without abundant embolized Neospirorchis eggs & 29.7 & $28.9-32.0$ & 1.19 & $1.10-1.34$ & 1.06 & $0.61-1.54$ \\
\hline
\end{tabular}


in robust nutritional condition were sought whenever possible to avoid the bias and confounding health issues that limit interpretation of findings in chronically ill animals, which comprise a large proportion of sea turtles that are recovered in a postmortem condition suitable for complete necropsy. Thus, the design and execution of this study tempered examination of a representative subset of animals with pursuit of fundamental questions regarding spirorchiidiasis in Florida sea turtles.

Three major features of the study population and categorization of data require discussion with regard to rationale and implications on data interpretation. First, most CM died during a single hypothermic stunning event, which provided 30/59 CM examined and $30 / 37$ of those in robust nutritional condition at stranding. Although inclusion of these turtles biased the total study population, the event provided an opportunity to examine a relatively large number of CM with a known, acute cause of death and was insightful in interpreting the significance of spirorchiidiasis in CM. However, as outlined in the following discussion, this bias must be considered when comparing these data to other size classes, as well as to turtles from other regions. Second, the use of an intermediate nutritional condition category was arguably a very subjective classification because it included turtles ranging from mildly underweight to visibly thin, but not emaciated. However, the necropsy and parasitological data support this categorization. Most turtles included in the intermediate condition category had singular identifiable causes of death, as did those in robust condition, in contrast to those emaciated turtles with advanced chronic disease. Furthermore, in the various aspects of spirorchiidiasis examined, data for turtles in the intermediate category often fell between statistically significant differences observed between robust and emaciated turtles, as expected in a continuum of disease relationships. Lastly, the bias produced by proportionately more robust adult CC may have confounded some of the statistical analyses, specifically the differences noted among size classes of turtles with thymic and enteric spirorchiidiasis. In both instances, proportionately more turtles in the smaller size categories were infected as compared to adult turtles; however, there were also more infected emaciated turtles as compared to animals that died in robust condition. Thus, the differences noted in the size class of infected turtles actually may have reflected the greater proportion of emaciated smaller turtles. The primary reason for the bias towards robust adult turtles in both CC and $\mathrm{CM}$ was traumatic deaths of adults during the breeding and nesting season.

Assessment of the significance of spirorchiidiasis in the study population was one of the primary objec- tives of this study. The SIR was devised as a conservative means for estimating significance and distinguishing pathological lesions of concern (but of unknown significance) from those in which a causal role in death and stranding could be reasonably inferred based on necropsy and other data. In $\mathrm{CC}$, spirorchiidiasis was interpreted as contributory to death or causal in $13.0 \%$ (7/54) of examined animals, and was a finding of concern, but of unknown significance, in an additional $33.3 \%(18 / 54)$. Of the latter group, it is notable that over half $(10 / 18)$ of those animals were in the robust or intermediate nutritional categories. Thus, relatively robust turtles with unrelated causes of death had pathological lesions of concern based on severity. In CM, spirorchiidiasis was found to be contributory to death in only 1 case $(2.7 \%$, $1 / 37$ ), and no examples of fatal spirorchiidiasis were observed. However, the proportion of CM in which the significance of the spirorchiidiasis could not be determined was comparable to the same for $\mathrm{CC}$, and most (9/10) CM in this category died of acute insults. These findings warrant comparison with the only published large study of spirorchiid-associated pathological lesions in sea turtles, which examined CM stranded in Moreton Bay, Queensland, Australia (Gordon et al. 1998). Gordon et al. (1998) reported spirorchiidiasis as the cause of death or a contributory cause of death in $10.4 \%(10 / 96)$ and $30.2 \%(29 / 96)$ of stranded turtles, respectively, which is much greater than was observed in Florida turtles, especially CM. Potential explanations for the disparity in these findings include demographic and geographic differences in the study populations, the criteria for interpreting significance, and health status at the time of stranding. Unfortunately, the size classes of examined animals were not given in the Australian study. In addition, the criteria for determining spirorchiidiasis as the cause of death were not specifically stated, which also limits comparisons between studies. The other significant difference between the Moreton Bay CM study and the present study is that many of the Australian turtles appear to have been chronically ill, whereas a significant number (54/104; Category 1 and 2) of Florida turtles died of acute insults. If examination had been limited to chronically ill (emaciated) CC (CM were underrepresented), the proportions of causal $(5.0 \%, 1 / 20)$ and contributory $(25.0 \%, 5 / 20)$ relationships are similar to the Australian CM study. In recognition of the caveats considered when examining chronically ill animals, Gordon et al. (1998) stated that further examination of turtles with acute illness is necessary to better define incidental or 'harmless,' i.e. background, infections and lesions.

Another previous study that bears comparison was an investigation of the epizootiology of spirorchiidiasis 
in Hawaiian CM that examined splenic egg counts (Work et al. 2005). This study found that splenic egg density was inversely related to both SCL and BCI. These trends contrast with some of the findings of the present study in that some of the parameters measured, viz. neurospirorchiidiasis and endarteritis, were greater in larger turtles. There are several factors that must be considered in comparing these studies. First, as noted by investigators in the Hawaiian study, splenic egg density likely reflects chronic accumulation and may not correlate with intensity of adult parasite infection, whereas the present study documented active infections with lesions associated with adult spirorchiids. Second, there appear to be differences between the spirorchiid fauna of Hawaiian CM and Florida CM and CC, the most significant of which is the apparent absence of Neospirorchis species in Hawaii. Neospirorchis was the most commonly detected genus in both CC and CM in Florida, and splenic egg density may not accurately reflect the selective accumulation of Neospirorchis eggs at various anatomic sites. Minimally, this technique would be difficult to validate for use in Florida turtles given the spectra of lesions and sites parasitized by Neospirorchis in both CC and CM.

Several pathological lesions of concern associated with spirorchiids were documented in Florida CC and CM. These observations provided further information on recognized problems, such as neurospirorchiidiasis, and identified new or poorly documented health issues, such as major arterial disease and spirorchiidassociated thyroiditis. It is not prudent to disregard these findings as insignificant or merely incidental, despite the fact that many examples were documented in turtles that died of apparently unrelated acute problems. Rather, the lesion severity and potential for physiological effects or sequelae suggest a possible negative impact on host fitness. Furthermore, infection and severe associated lesions in relatively robust turtles suggest that spirorchiids can act as primary pathogens, i.e. they have the ability to produce injury in otherwise healthy individuals, as opposed to a more limited role as opportunistic agents in debilitated animals. Conversely, these otherwise robust and, in many cases, actively foraging infected turtles with pathological lesions provide an important context in which to interpret similar findings in chronically ill animals with other confounding health issues. Thus, based on the findings of this study, spirorchiids are regarded as potential primary pathogens and a health concern in free-ranging turtles, but interpretation of significance in individual cases requires caution, especially with regard to concluding the cause of death.

The high prevalence of major arterial disease primarily associated with Hapalotrema mistroides in
$\mathrm{CC}$ was notable and underscores the importance of a complete examination of the aortic system and larger arteries when performing necropsies. There were no significant differences among nutritional condition at stranding categories, and severe lesions were observed in turtles that died of acute traumatic injuries and brevetoxicosis. Also, significantly more adult CC were infected by Hapalotrema sp. (with associated arteritis), which suggests that age-related factors, such as exposure over time or differences in exposure to intermediate host(s), influences infection. The occurrence and significance of Hapalotrema-associated arteritis may be underestimated because adult CC comprised a small proportion of the stranded sea turtles.

Although different identifiable spirorchiid species were found in CC as compared to CM, a number of similarities were seen between turtle species in this study. Three of 4 adult CM examined had severe arterial disease associated with Learedius learedi and Hapalotrema postorchis. In contrast, infection by $L$. learedi was only observed in 1 of 46 examined immature $\mathrm{CM}$, and very few parasites and no significant lesions were present in this single case. No Hapalotrema were found in the immature CM examined. Thus, Learedius and Hapalotrema appeared to be more of a health issue in adult CM in Florida waters. This observation is consistent with a parasitological survey of nesting female CM in Tortuguero, Costa Rica, in which L. learedi was found in 39 of 40 of adult turtles (Santoro et al. 2006).

There were some notable differences in the arterial lesions observed in the present study and those described by Gordon et al. (1998). Both studies observed lesions in the heart and major arteries in significant numbers of stranded sea turtles. Although different spirorchiid species were identified, the left aorta was the most common site of both lesions and localization of some adult spirorchiids (Hapalotrema species in the present study). Gordon et al. (1998) described arterial thrombus formation, mural and occlusive, in 81/96 turtles, and proposed an interesting process of 'exteriorization' in which thrombi resolved by migrating from the vascular lumen through the arterial wall. In contrast, in Florida sea turtles, mural thrombus formation in Category 1 necropsies was only observed in less than half of affected turtles (10/24), and only one of these involved a partially occluding luminal thrombus. Part of the discrepancy may lie in use of the term 'thrombus.' Gordon et al. (1998) included descriptions of spirorchiid eggs and pigmented debris, some of which appeared to be involved in the exteriorization process, in their definition of thrombi. In our study, we limited the use of the term thrombus to lesions characterized by the formation of fibrinocellular clots on the 
endothelial surface. The exteriorization process, as described by Gordon et al. (1998), was not observed, although the inflammation was transmural in 15/24 cases examined histologically, and there were granulomas containing spirorchiid eggs or pigmented debris observed within these lesions. In a few cases, the impression of an exteriorization process could be claimed. In addition to semantics, some of the variation in described lesions could reflect differences in lesions associated with different species of spirorchiids. Specific identification was only performed for a small subset of turtles in the Australian study, and identified species in the heart and major vessels included $H$. pambanensis and $H$. postorchis. There was only a single example of arteritis associated with $H$. postorchis infection in the current study population, and severe lesions were more often associated with $H$. mistroides infection in CC.

Although we did not observe the large pigmented thrombi as described by Gordon et al. (1998), an interesting pattern of degeneration and necrosis of the tunica media was seen in $7 / 24$ Category 1 cases. It was remarkable that sequelae, such as thrombosis, aneurysm formation, or rupture, were not observed more frequently given the severity of some of these lesions. To our knowledge, this pattern of medial necrosis has not been previously described in spirorchiid-associated lesions.

Neurospirorchiidiasis (Neospirorchis sp.) has been a concern in CC in Florida since being implicated in an MME in south Florida in 2000 and 2001 (Jacobson et al. 2006). The findings of the present study have identified several features of neurological spirorchiid infection in $\mathrm{CC}$ that are critical to future disease studies and provide better understanding of the host-parasite relationship. First, neurospirorchiidiasis was observed in $51.7 \%$ of the CC (46/89), of which $64.4 \%$ (29/45) were characterized as low intensity (Grade 1) infections. Over $11.1 \%(5 / 45)$ of cases, however, were intense infections (Grade 3). There are anecdotal reports from sea turtle veterinarians and rehabilitators of improvement of vague neurological symptoms following antihelminthic therapy; however, correlative clinical studies with confirmation of infection are lacking, primarily due to lack of methods for specific antemortem diagnosis. Unfortunately, the findings of this study did not clarify the clinical or health significance of neurospirorchiidiasis. Of the 5 turtles with severe (Grade 3) neurospirorchiidiasis, all were either found dead or died soon after discovery and did not receive a neurological examination. Three of these animals were severely emaciated with multiple disease processes, including spirorchiid-associated lesions in other organs. Of the remaining 2 cases, 1 died of brevetoxicosis, and thus would have exhibited confounding neurological symptoms, and the other died of a gastrointestinal perforation and secondary coelomitis. Therefore, of these 5 cases, it can only be stated for a single turtle that abundant meningeal spirorchiids were observed, but were unrelated to cause of death based on good nutritional condition and known cause of death (gastrointestinal perforation). Of the 44 brains examined with neurospirorchiidiasis, no associated hemorrhagic or ischemic lesions were identified.

Another notable finding was the significant relationship between neurospirorchiidiasis and adult CC. As with Hapalotrema infections, this observation may reflect increasing probability of exposure over time, selective exposure, or other age-related factors. An age-dependent effect is relevant to the 2000 and 2001 CC mortality event in south Florida (Jacobson et al. 2006). At the time of the investigation of this event and in lieu of true prevalence data, the intensity of neurospirorchiidiasis in the effected $\mathrm{CC}$ was determined by using unrelated cases to establish baseline infections. These control cases were primarily stranded CC from northeastern Florida. In contrast to the $\mathrm{CC}$ from the mortality event in south Florida, which had a median SCL of $80.0 \mathrm{~cm}$ (A. M. Foley unpubl. data excluding 1 unusually small $\mathrm{CC}$ ), the median SCL of the control CC was $61.5 \mathrm{~cm}$. In retrospect, the control cases used in the investigation of the 2000/2001 south Florida mortality event were inappropriate given the lower prevalence of neurospirorchiidiasis in small CC. Thus, association between neurospirorchiidiasis and the 2000/2001 MME cannot be concluded based on this comparison.

Neurological Neospirorchis infections were not limited to CC, but were also observed in CM. Similar to $\mathrm{CC}$, there appears to be a size relationship in stranded Florida CM as well. All infected turtles with adult parasites were adult or large subadult turtles. Neurospirorchiidiasis was not found in any of the smaller turtles, with only 2 possible exceptions in which eggs, but no adult parasites, were observed. No infected CM had high intensity neurological infections. Neurospirorchiidiasis has been previously documented or depicted in photographs of CM in at least 3 previous studies (Glazebrook et al. 1989, Gordon et al. 1998, Raidal et al. 1998), although the genus appears to have been correctly identified in only 1 report: Gordon et al. (1998) described microscopic flukes, consistent with Neospirorchis sp., in the meninges of $73.6 \%$ (53/72) of examined CM. The species N. schistosomatoides was identified in 2 of these cases.

Another pathological finding of concern in both species was parasitism of the thyroid gland, which resulted in extensive glandular injury in severe cases. The thyroid gland was one of the most common sites inhabited by spirorchiids in both CC and CM. Compa- 
rable proportions of the different age/size classes were infected. There were significantly more infections and more intense infections in emaciated CC. The same comparisons were not possible for $\mathrm{CM}$; however, 2 cases of Grade 3 lesions were observed in relatively robust CM that died of hypothermia. Furthermore, there was no evidence of any significant effect on BCI in the immature CM that died of hypothermia that were observed to have intra-thyroidal Neospirorchis. The significance of thyroid injury associated with Neospirorchis infection requires further study.

An interesting difference in parasitism of endocrine organs by Neospirorchis sp. in CC and CM was that adult Neospirorchis were observed in adrenal gland vasculature of $32.0 \%$ (16/50) of Category 1 and 2 CC but in none of the 50 examined CM. Gordon et al. (1998) occasionally observed 'microscopic' flukes (presumably Neospirorchis sp.) in adrenal glands of CM; however, this finding was not seen in the present study. Following the conclusion of this study, adult Neospirorchis sp. were observed in the adrenal gland of $1 \mathrm{CM}$ from Florida. The potential differences in the prevalence of adrenal gland infections between the 2 species of sea turtles could reflect differences in the Neospirorchis species involved or variation in hostparasite interaction.

Involvement of the thymus in spirorchiid infections, as observed in many $\mathrm{CC}$, has not been described in the literature. Notably, this finding was not seen in CM, although infection of the thyroid gland, which often was concurrent with thymic parasitism in $\mathrm{CC}$, was observed in both species.

The organ system most commonly involved in spirorchiidiasis (both egg embolization and the occurrence of adult spirorchiids) was the gastrointestinal tract. Again, differences were observed between CC and CM. Adult Neospirorchis sp. within the enteric submucosa and localized egg deposition characterized by large black, serpiginous egg masses were observed in over $65.8 \%$ (50/76) of CC. Interestingly, serpiginous Neospirorchis egg masses were not observed in the enterocolic submucosa of the CM in the present study, although similar egg masses have been described in CM in Australia (Gordon et al. 1998).

Proportionately more emaciated turtles had gastrointestinal Neospirorchis egg masses than robust turtles, and all severe (Grade 3 ) infections were in emaciated animals. In contrast to spirorchiid infections in other organ systems, proportionately more infected turtles with enteric involvement were large immature turtles (>65-85 cm SCL group), as compared to mature turtles, and most of the Grade 2 and higher infections were animals in this size class. This possible relationship with size class must be cautiously interpreted given the aforementioned bias in our study population.
As in the present study, Wolke et al. (1982) also described bacterial enteritis in CC associated with spirorchiid eggs, but they did not identify the spirorchiid type.

The other gastrointestinal lesions observed were those associated with Hapalotrema species in $\mathrm{CC}$, which ranged from relatively small numbers of diffusely embolized eggs and embolized egg masses within subserosal and submucosal vessels to more dense, transmural egg masses with associated ischemic lesions and ulceration of the stomach and intestine. One of these latter cases was the only confirmed example of fatal spirorchiidiasis. There may be differences in the enteric lesions associated with different Hapalotrema species. The egg masses in the CC with fatal spirorchiidiasis, which involved a probable novel species of Hapalotrema, were much more extensive than was observed in turtles infected with $H$. mistroides and $H$. pambanensis. Another $\mathrm{CC}$ that was also infected with the probable novel species of Haplotrema had similarly severe enteric lesions but was excluded from the study because examination was limited to the small intestine. A formal description of this probable new species will be reported elsewhere. Additional lesions in the digestive tract associated with Hapalotrema eggs included enteric polyp formation, similar to schistosomal polyposis of humans (Mesquita et al. 2003), and papillary lesions in the gall bladder secondary to granulomatous cholecystitis.

The testes were another organ in which significant pathological lesions were associated with parasitism by Neospirorchis species. Although only observed in 3 turtles, these cases comprised $25.0 \%$ (3/12) of examined adult male CC. There may have been persistent, negative effects on reproduction had these turtles recovered or been rehabilitated. This finding was not observed in the 3 adult male CM examined, nor was parasitism of the ovary or other aspects of the female reproductive system identified in either species.

In addition to pathological lesions associated with adult parasites and/or concentrated egg deposition at specific sites, diffuse embolization of eggs, especially to the lungs and gastrointestinal tract, was severe in a number of cases. With the exception of the aforementioned specific examples, most of the eggs were Neospirorchis species in both CC and CM. Multiorgan accumulation of eggs was a primary finding in 4/7 cases in which spirorchiidiasis was interpreted as contributory to the cause of death and was a lesion of concern in several turtles in the undetermined significance category. Interpretation of significance must be approached very cautiously, as noted in the group of CM that died from hypothermia. Six of these turtles were observed to have moderate or large numbers of Neospirorchis eggs embolized to the lungs and/or gas- 
trointestinal tract; most of these animals also had Neospirorchis adults and eggs in the thyroid gland. The significance of egg accumulation in these cases was unknown, which was reflected in the undetermined impact classification. There was no observable difference on nutritional condition as assessed by body weight, abundance of internal fat, and BCIs. However, effects may manifest when other confounding health problems, such as concurrent disease processes or environmental stressors, are present.

Spirorchiid infection (all species included) was observed in $96.4 \%(54 / 56)$ of CC and $92.0 \%$ (46/50) of $\mathrm{CM}$ examined in Category 1 and 2 necropsies. These numbers were comparable to previous studies that also used methodology intended to detect spirorchiid infection (Dailey et al. 1992, Gordon et al. 1998, Work et al. 2005, Santoro et al. 2006). Infection by multiple genera was frequently observed in CC and all adult CM, but rarely in immature CM. Some of the spirorchiid species identified in this study were documented for the first time in the Western North Atlantic region. Species reported in Florida prior to 2004 included Carettacola bipora (Manter \& Larson 1950), Hapalotrema synorchis (Luhman 1935), and Neospirorchis pricei (Manter \& Larson 1950). The current study additionally documented $H$. mistroides and a probable novel Hapalotrema in CC, and $H$. postorchis and Learedius learedi in free-ranging $\mathrm{CM}$. The only spirorchiid species that was previously documented in Florida waters that was not observed in our sample group was $H$. synorchis. In addition, there is potential for unrecognized diversity in the genus Neospirorchis that could not be revealed by morphological examination due to the inability to collect intact specimens. There were obvious site predilections for adult Neospirorchis, including the leptomeninges, heart and major vessels, endocrine organs, thymus, and submucosa of the alimentary tract. Although intact adults could not be examined, there were distinct differences in size and general morphological features among Neospirorchis adults and eggs (data not shown) from different anatomic locations that hint at unrecognized diversity and justify further study. Lastly, as noted for C. hawaiiensis in Hawaiian CM, C. bipora in CC typically were observed in small numbers (both adults and eggs) and with minimal associated pathology other than occasional mild inflammation of hepatic vessels (Work et al. 2005).

Notably, the spirorchiids we were able to identify to species were only found in CC or CM. Several marine spirorchiids are known to parasitize multiple sea turtle species, and it has been generally stated that there is no evidence of any host specificity at the generic or species levels (Smith 1972, 1997b). At least 1 study, however, has offered evidence of potential host restric- tion in freshwater spirorchiids (Byrd 1939, cited by Goodman 1987). It must be considered that any apparent host restrictions in sea turtles in published reports could reflect bias, as most studies have only examined $\mathrm{CM}$. Only a few studies have examined both $\mathrm{CC}$ and $\mathrm{CM}$ inhabiting the same general region, and all examined either an unspecified number of or very few turtles (Looss 1902, Simha \& Chattopadhyaya 1980, Platt \& Blair 1998). Thus far, Learedius learedi has only been described in CM and hawksbill turtles Eretmochelys imbricata, and Hapalotrema postorchis has only been described in CM (Smith 1972, 1997a). Neither of these spirorchiid species was observed in CC in the current study; thus, infection may be rare or absent in this species, at least in this region. H. pambanensis has only been reported in CM and E. imbricata (Smith 1997a). The current study has now added CC to the list of known hosts for this species. None of the specimens of $H$. mistroides or the probable novel Hapalotrema were collected from $\mathrm{CM}_{\text {; }}$ however, examination of additional larger $\mathrm{CM}$ is necessary given the apparent rarity of Hapalotrema species in smaller immature CM in Florida waters. Lastly, the single species of Carettacola, C. bipora, was limited to CC, which was the identified host in the only report and original description of this species (Manter \& Larson 1950). Some specialization or host restriction of marine spirorchiids in sea turtles may well occur but requires further investigation. Results from the present study minimally suggest that there may be large differences in the regional prevalence of species of spirorchiids in CC and CM. Differences in parasite fauna would not be surprising given the differences in diet and habitat utilization between these 2 sea turtle species.

The somewhat regular distribution of spirorchiid adults and eggs may reflect some degree of microhabitat selectivity. There are reports of both host-organ specificity (Wall 1951), as well as lack of specific tropisms in the freshwater spirorchiid literature (Holliman et al. 1971, Platt 1993). It is reasonable to hypothesize that specialization would evolve, in at least some circumstances, as it has in mammalian and avian schistosomes (Marquardt et al. 2000). Although this issue will require additional techniques, e.g. molecular characterization, to further study Neospirorchis species, there is evidence of site tropism in the more easily identified spirorchiids with linguiform morphology. For example, all reports of Carettacola species in which anatomic location is specified have involved adult parasites collected from hepatic blood vessels (Dailey et al. 1991, 1992, Graczyk et al. 1995). Similarly, all adult C. bipora in the present study were collected from either hepatic vessels or mesenteric vessels, the latter of which may have reflected presence of parasites in extrahepatic portal circulation. The 3 Hapalotrema species ob- 
served primarily in the heart and major arteries of the sea turtles in this study were also observed in the same areas in a previous study (Smith 1997a). As in the present study, both $H$. mistroides and Learedius learedi also have been reported in hepatic circulation (Smith 1997a, Santoro et al. 2006). Although adult spirorchiids have been collected from these various vessels, there appears to be a predilection for the left aorta in some Hapalotrema species, as observed by Gordon et al. (1998) and for $H$. mistroides and $H$. postorchis as observed in the current study. The left aorta is the major conduit to the superior mesenteric artery, and thus provides access to smaller enteric vessels leading to the mucosa through which eggs may exit to the external environment.

Microhabitat utilization is relevant both to potential health effects in the turtle host as well as the life cycle of the parasite. The strategy for propagation of the spirorchiid life cycle is unclear for sites where the likelihood of eggs exiting to the external environment is seemingly remote, such as the brain or thyroid gland. Holliman et al. (1971) suggested that the death of hyperinfected turtles may contribute to the parasite life cycle by releasing eggs entrapped within internal tissues. In sea turtles examined in the present study, live miracidia were observed in spirorchiid eggs following postmortem intervals of 48 to $72 \mathrm{~h}$, which is ample time for dispersal of a carcass by marine scavengers. Another possibility is that a given sea turtle species may be an aberrant host, such as in cases of neurospirorchiidiasis in CC. However, the high prevalence and apparent incidental nature of most infections, as observed in this study, would be an unusual presentation of an aberrant host. Alternatively, the numbers of eggs that reach the lungs and/or gastrointestinal tract over time may be sufficient for shedding.

\section{CONCLUSIONS}

Spirorchiid trematodes and their associated cardiovascular lesions are among the oldest disease-related observations in the sea turtle literature (Looss 1902). Over the last $100 \mathrm{yr}$, significant progress has been made in the characterization of these parasites. However, fundamental gaps remain in our understanding of their impact on sea turtle health. Our ability to study diseases of sea turtles is limited by the difficulty in obtaining fresh carcasses, the investment required for thorough postmortem examinations, and the advanced chronic state of disease that typifies many of the animals that are necropsied.

Prevalence of spirorchiid infection was high in examined $\mathrm{CC}$ and $\mathrm{CM}$ and was comparable to that observed by other investigators using similar method- ologies. The proportion of turtles of both species, especially adults, with spirorchiid-associated cardiovascular lesions of concern was notable. Necropsies of several nutritionally robust turtles that died from acute trauma revealed that these parasites have the ability to infect and produce severe lesions in seemingly healthy turtles. Given the extent of some lesions and the apparent diminished integrity of affected arteries, we were surprised that more examples of fatal sequelae were not observed as was more often the case in previous studies. However, many sea turtles that develop fatal complications of this sort may die at sea and fail to be well-represented as strandings.

The findings of this study increased the number of species of spirorchiids known to parasitize Florida sea turtles, the spectrum of organs in these sea turtles known to be infected by spirorchiids, and the types of pathological lesions known to be associated with these spirorchiid infections. Many questions regarding the health implications of spirorchiidiasis remain. The effects of neurological infections and parasitism of endocrine organs, especially the thyroid gland, require further investigation. The prevalence data and size class relationships identified herein will hopefully be used to guide and facilitate such studies. In addition, we encourage the recognition of the many distinct forms of spirorchiidiasis in future studies as opposed to simply lumping these many manifestations (and thus representing a wide range of potential effects) under the single general category of spirorchiid infection.

Acknowledgements. We thank K. Minch, R. Bailey, S. Schaf, E. DeMaye, K. Sonderman, N. Brewer, STSSN participants, and staff at the sea turtle rehabilitation facilities, especially $\mathrm{N}$. Metee, S. Fournies, and M. Bauer, in Florida for their invaluable assistance retrieving and transporting stranded sea turtles. We also thank A. McIntosh for help with parasitological collections and examinations and J. Dame and R. Alleman for their valuable input. Partial funding was provided by Awards 2003-0206-011 administered by the National Fish and Wildlife Foundation and NA06NMF4720005 administered by the National Oceanic and Atmospheric Administration, and by grant number 06-017R from the Florida Sea Turtle Tag Grants program.

\section{LITERATURE CITED}

Bjorndal KA, Bolten AB, Chaloupka MY (2000) Green turtle somatic growth model: evidence for density dependence. Ecol Appl 10:269-282

Dailey MD, Fast ML, Balazs GH (1991) Carettacola hawaiiensis n. sp. (Trematoda: Spirorchidae) from the green turtle, Chelonia mydas, in Hawaii. J Parasitol 77:906-909

Dailey MD, Fast ML, Balazs GH (1992) A survey of the Trematoda (Platyhelminthes: Digenea) parasitic in green turtles, Chelonia mydas (L.) from Hawaii. Bull South Calif Acad Sci 91:84-91 
Foley AM, Schroeder BA, Redlow AE, Fick-Child KJ, Teas WG (2005) Fibropapillomatosis in stranded green turtles (Chelonia mydas) from the eastern United States (1980-98): trends and associations with environmental factors. J Wildl Dis 41:29-41

Foley AM, Minch KS, Dutton PH, Summers TM, Redlow AE, Lessman J (2007) Characteristics of a green turtle (Chelonia mydas) assemblage in northwestern Florida determined during a hypothermic stunning event. Gulf Mex Sci 2:131-143

Glazebrook JS, Campbell RS, Blair D (1989) Studies on cardiovascular fluke (Digenea: Spirorchiidae) infections in sea turtles from the Great Barrier Reef, Queensland, Australia. J Comp Pathol 101:231-250

Goodman JD (1987) A new blood fluke, Hapalorhynchus beadlei n. sp. (Spirorchiidae), and a note on Allossostomoides [sic] (Paramphistomistomidae), in Pelusios williamsi lutescens from Uganda. Trans Am Microsc Soc 106:80-84

> Gordon AN, Kelly WR, Cribb TH (1998) Lesions caused by cardiovascular flukes (Digenea: Spirorchidae) in stranded green turtles (Chelonia mydas). Vet Pathol 35:21-30

Graczyk TK, Aguirre AA, Balazs GH (1995) Detection by ELISA of circulating anti-blood fluke (Carettacola, Hapalotrema, and Learedius) immunoglobulins in Hawaiian green turtles (Chelonia mydas). J Parasitol 81: 416-421

Herbst LH (1994) Fibropapillomatosis of marine turtles. Annu Rev Fish Dis 4:389-425

Herbst L, Ene A, Su M, Desalle R, Lenz J (2004) Tumor outbreaks in marine turtles are not due to recent herpesvirus mutations. Curr Biol 14:R697-R699

Holliman RB, Fisher JE, Parker JC (1971) Studies on Spirorchis parvus (Stunkard, 1923) and its pathological effects on Chrysemys picta picta. J Parasitol 57:71-77

Jacobson ER, Homer BL, Stacy BA, Greiner EC and others (2006) Neurological disease in wild loggerhead sea turtles Caretta caretta. Dis Aquat Org 70:139-154

Looss A (1902) Über neue und bekannte Trematoden aus Seeschildkröten. Nebst Erörterungen zur Systematik und Nomenclatur. Zool Jahrb Abt Syst Oekol Geogr Tiere 16:411-894

Luhman M (1935) Two new trematodes from the loggerhead turtle (Caretta caretta). J Parasitol 21:274-276

Manter HW, Larson MI (1950) Two new blood flukes from a marine turtle, Caretta caretta. J Parasitol 36:595-599

Marquardt WC, Demaree RS, Grieve RB (2000) Parasitology and vector biology, 2nd edn. Academic Press, San Diego, CA

McMichael E (2005) Ecology of juvenile green turtles (Chelonia mydas) at a temperate foraging area in the northeast- ern Gulf of Mexico. MSc thesis, University of Florida, Gainesville, FL

Mesquita NF, Silva RA, Brandão CL, Dinis-Ribeiro MJ, Fernandes NS, Lomba-Viana H, Dias LM (2003) Schistosomal colonic polyposis. Gastrointest Endosc 58:910-911

Platt TR (1993) Taxonomic revision of Spirorchis MacCallum, 1919 (Digenea: Spirorchidae. J Parasitol 79:337-346

Platt TR (2002) Family Spirorchiidae Stunkard, 1921. In: Gibson DI, Jones A, Bray RA (eds) Keys to Trematoda Vol 1. CABI Publishing, New York, p 453-467

Platt TR, Blair D (1998) Redescription of Hapalotrema mistroides (Monticelli, 1896) and Hapalotrema synorchus Luhman, 1935 (Digenea: Spirorchidae), with comments on other species in the genus. J Parasitol 84:594-600

Raidal SR, Ohara M, Hobbs RP, Prince RI (1998) Gram-negative bacterial infections and cardiovascular parasitism in green sea turtles (Chelonia mydas). Aust Vet J 76:415-417

Santoro M, Greiner EC, Morales JA, Rodríguez-Ortíz B (2006) Digenetic trematode community in nesting green sea turtles (Chelonia mydas) from Tortuguero National Park, Costa Rica. J Parasitol 92:1202-1206

Simha SS, Chattopadhyaya DR (1980) Studies on the trematode parasites of reptiles found in India. Contribution to the knowledge of blood flukes from marine turtles, from the Gulf of Manar [sic], South India. J Zool Soc India 30: 69-82

Smith JW (1972) The blood flukes (Digenea: Sanguinicolidae and Spirorchidae) of cold-blooded vertebrates and some comparison with the schistosomes. Helminthol Abstr Ser A 41:161-194

Smith JW (1997a) The blood flukes (Digenea: Sanguinicolidae and Spirorchidae) of cold-blooded vertebrates: part 2. Appendix I: comprehensive parasite-host list; Appendix II: comprehensive host-parasite list. Helminthol Abstr 66:329-344

Smith JW (1997b) The blood flukes (Digenea: Sanguinicolidae and Spirorchidae) of cold-blooded vertebrates: part 1. A review of literature published since 1971, and bibliography. Helminthol Abstr 66:255-294

Wall LD (1951) The life history of Vasotrema robustum (Stunkard, 1928), Trematoda: Spirorchidae. Trans Am Microsc Soc 70:173-184

Witherington BE, Erhart LM (1989) Hypothermic stunning and mortality of marine turtles in the Indian River Lagoon system, Florida. Copeia 1989:696-703

- Wolke RE, Brooks DR, George A (1982) Spirorchidiasis in loggerhead sea turtles (Caretta caretta): pathology. J Wildl Dis 18:175-185

Work TM, Balazs GH, Schumacher JL, Amarisa M (2005) Epizootiology of spirorchiid infection in green turtles (Chelonia mydas) in Hawaii. J Parasitol 91:871-87 
Appendix 1. Criteria for determining spirorchiid trematode impact rating

\begin{tabular}{|c|c|}
\hline Score & Definition and criteria \\
\hline 1 & None detected. \\
\hline 2 & $\begin{array}{l}\text { Incidental infection. Numbers of adult spirorchiids, if detected, are low }(\leq 4) \text { and egg deposition in tissues consists of } \\
\text { rare individual embolized eggs and/or isolated egg masses. Associated tissue/organ injury or inflammation is mild } \\
\text { and focal in distribution. }\end{array}$ \\
\hline 3 & $\begin{array}{l}\text { Contributory. Large numbers of adult spirorchiids and/or eggs are associated with significant organ injury. Included } \\
\text { in this category are turtles in which severe, multisystemic spirorchiid egg embolization is one of multiple significant } \\
\text { pathological findings. Also included are turtles in which injury/inflammation associated with spirorchiids has } \\
\text { resulted in or exacerbated other conditions, including thrombosis and secondary bacterial infection. }\end{array}$ \\
\hline 4 & $\begin{array}{l}\text { Fatal. The severity and extent of organ/tissue injury or inflammation associated with spirorchiid adults or eggs } \\
\text { supports spirorchiidiasis as the cause of death. }\end{array}$ \\
\hline 5 & $\begin{array}{l}\text { Unknown. Large numbers of adult spirorchiids and/or eggs are present and/or there is significant associated organ } \\
\text { injury, but significance cannot be confidently determined from necropsy data. Included in this group are turtles with } \\
\text { a known cause of death in which association, if any, with spirorchiidiasis is unknown. }\end{array}$ \\
\hline
\end{tabular}

Appendix 2. Criteria for grading spirorchiid infection and associated pathological lesions

\section{Grade Criteria}

\section{Arteritis of large arteries associated with Hapalotrema infection}

$0 \quad$ No gross lesions

Histological correlate: within normal limits

$1 \quad$ Focal gross lesion $(\leq 1.5 \mathrm{~cm}$ diameter $)$

Histological correlate: minimal or mild subintimal inflammatory infiltrate and/or fibrosis

2 Focally extensive $(1.5 \mathrm{~cm}$ to $<4.0 \mathrm{~cm}$ in greatest dimension) OR multifocal: maximum of 3 lesions (any single lesion $<2.0 \mathrm{~cm}$ diameter or $<4.0 \mathrm{~cm}$ in greatest dimension)

Histological correlate: moderate changes including inflammation and intimal proliferation

3 Regionally extensive (single or multiple, $>4.0 \mathrm{~cm}$ in any dimension) OR diffuse lesions involving 1 or more vessels Histological correlate: changes as under 2 but severe and/or any of the following: thrombus formation, aneurysm formation, necrosis of tunica media

Neurospirorchiidiasis (Neospirorchis species)

$0 \quad$ No egg masses or adults / individual embolized eggs only

1 Small numbers: typically 1 to 5 egg masses measuring $<3 \mathrm{~mm}$ in greatest dimension and/or small numbers (1 to 3 ) adults

2 Moderate numbers: typically 5 to 10 egg masses with most measuring $<3 \mathrm{~mm}$ in greatest dimension and/or 1 to 2 larger coalescing egg masses distending meningeal vessels

3 Large numbers: $>10$ egg masses with regionally intense or diffuse distribution and/or $>2$ larger coalescing egg masses distending meningeal vessels

Parasitism of the thyroid gland and thymus (Neospirorchis species)

$0 \quad$ No eggs OR rare to small numbers of embolized eggs only

1 Small numbers of adults and/or egg masses

$2 \quad$ Moderate numbers (includes focally extensive aggregates and diffuse lesions) and/or adults

3 Large numbers (large areas of gland/thymus effaced by eggs and associated inflammation)

Enteric spirorchiidiasis (Neospirorchis species)

$0 \quad$ No egg masses

$1 \quad 1$ to 10 distinct egg mass

$2>10$ to 50 distinct egg masses

$3>50$ distinct egg masses

Editorial responsibility: Alex Hyatt, Geelong, Victoria, Australia
Submitted: July 15, 2009; Accepted: January 6, 2010

Proofs received from author(s): March 10, 2010 